\section{Dal castrum altomedievale al restauro settecentesco: storia e archeologia del castello di Sorrivoli (Roncofreddo - FC)}

\section{From the early medieval castrum to the eighteenth- century restoration: history and archaeology of the castle of Sorrivoli (Roncofreddo - FC)}

Andrea Fiorini*

Dipartimento di Archeologia, Università di Bologna (Italia)

\section{Riassunto}

The methods and tools used to determine the history of construction and building techniques of the castle of Sorrivoli (Roncofreddo - FC) are here described. This is a research project carried out within a study coordinated by the teaching of Medieval Archaeology (University of Bologna), which has the purpose of conducting a census of all the medieval castles in Romagna. There is certain news from the tenth century concerning the castle to be studied. However, the investigations have shown that the standing preserved structures must be dated from the fourteenth century onwards. The system of photogrammetric survey of the walls and the technique of threedimensional modelling of the structures are here described. An extended part of this paper is also devoted to results of stratigraphic, typological, geological, metrological and comparative mensio-cronological analysis.

Parole chiave: Romagna; castello; tecniche costruttive; metrologia; fotogrammetria; 3D.

\section{Abstract \\ The methods and tools used to determine the history of construction and building techniques of the castle of Sorrivoli (Roncofredo - FC) are here described. This is a research project carried out within a study coordinated by the teaching of Medieval Archaeology (University of Bologna), which has the purpose of conducting a census of all the medieval castles in Romagna. There is certain news from the tenth century concerning the castle to be studied. However, the investigations have shown that the standing preserved structures must be dated from the fourteenth century onwards. The system of photogrammetric survey of the walls and the technique of three- dimensional modelling of the structures are here described. An extended part of this paper is also devoted to results of stratigraphic, typological, geological, metrological and comparative mensio-cronological analysis.}

Key words: Romagna; castle; building techniques; metrology; photogrammetry; 3D.

*andrea.fiorini6@unibo.it

\section{INTRODUZIONE}

Da qualche anno a questa parte l'insegnamento di Archeologia Medievale dell'Università di Bologna (Facoltà di Conservazione dei Beni Culturali, sede di Ravenna), ha in corso un progetto di studio dei castelli medievali dell'Emilia-Romagna, in collaborazione con il Dipartimento di Medievistica della stessa Università e con l'IBC (Istituto per i beni artistici culturali e naturali della Regione EmiliaRomagna). Si tratta di un progetto di schedatura di tutti i castelli della regione e dei loro resti archeologici, che coinvolge sia i siti abbandonati che quelli a continuità di vita (Augenti et alii, 2010).

$\mathrm{Nel}$ corso di questo progetto, che ha come scopo il censimento generale delle evidenze, è stato già possibile avviare alcuni sottoprogetti concepiti per approfondire lo studio di alcuni monumenti particolarmente interessanti (fig. 1). Si tratta sia di indagini volte ad indagare nel dettaglio i resti strutturali ancora visibili (qualora particolarmente meritevoli, come nel caso del castello di Meldola - FC), sia di indagini di scavo (come nel caso del castello di Rontana presso Brisighella - RA), sia, talvolta, di entrambe le cose (come nel caso del castello di Zena - PC).

Nell'ambito di questo progetto di censimento globale delle fortificazioni romagnole si è lanciata una serie di indagini finalizzate alla determinazione della storia costruttiva del castello di Sorrivoli (Roncofreddo - FC), un complesso architettonico pluristratificato di particolare interesse giacché esemplifica chiaramente uno dei processi evolutivi più diffusi dei siti fortificati romagnoli: in età moderna le rocche tardomedievali, allestite sulle vestigia dei castra altomedievali, vengono riconvertite in comodi palazzi residenziali (fig. 2) ${ }^{1}$.

Sorrivoli è una località del basso Appennino, situata nel comune di Roncofreddo e appartenente alla provincia di Forlì-Cesena (Emilia-Romagna). Da Sorrivoli i primi centri abitati che si incontrano sono Ardiano (a SudOvest), Diolaguardia (a Ovest), Montereale (a NordOvest), Saiano (a Nord), Carpineta (a Nord-Est), Casale (a

\footnotetext{
${ }^{1}$ Il castello di Sorrivoli è incluso come argomento nella tesi di laurea di Alberto Urcia (Urcia, 2009) e nella tesi di dottorato di chi scrive (Fiorini, 2010b). Nel presente articolo si descrivono, in sintesi, le attività di ricerca coordinate dal Laboratorio di Archeologia dell'Architettura del Dipartimento di Archeologia (direzione scientifica Andrea Augenti, responsabile operativo Andrea Fiorini) e condotte da Valentina Archetti (analisi dei canoni mensori), Andrea Fiorini (studio del contesto storico, analisi delle tecniche murarie, degli apparati decorativi, dei canoni mensori, delle fonti scritte, iconografiche e cartografiche), Enrica Giorgioni (analisi mensiocronologica dei laterizi), Emanuela Maroni (studio tipologico delle aperture, analisi stratigrafica e delle tecniche murarie), Alberto Urcia (rilievo e trattamento informatico dei dati) e Fabio Zaffagnini (analisi dei litotipi e identificazione dei luoghi di provenienza del materiale costruttivo). Per una relazione estesa v. Fiorini, c.s.
} 


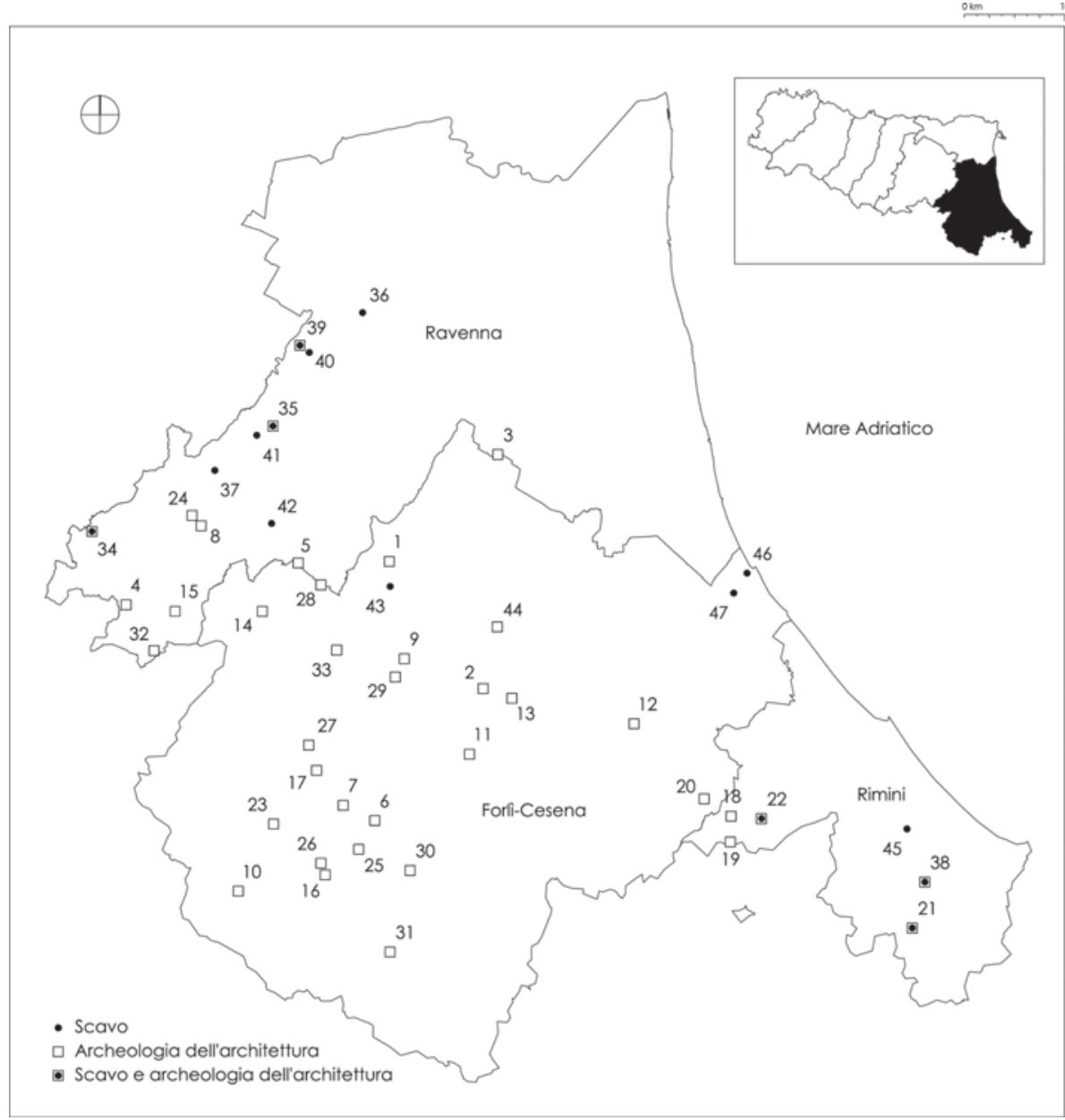

Fig. 1. Le indagini sui castelli in Romagna: scavi e archeologia dell'architettura (da Augenti et alii, 2010, p. 62, fig. 1).
Est) e Monteleone (a Sud). Il castello si trova sulla sommità di una collina, a 268 metri sul livello del mare, distante circa 10 chilometri dalla città di Cesena e circa 700 metri dal torrente Pisciatello (fig. 3). Attorno al castello il paesaggio collinare è caratterizzato dalla presenza di vasti terreni agricoli (seminativi, frutteti, vigneti e pioppeti) e boschi a prevalenza di querce, carpini e castagni (fig. 4).

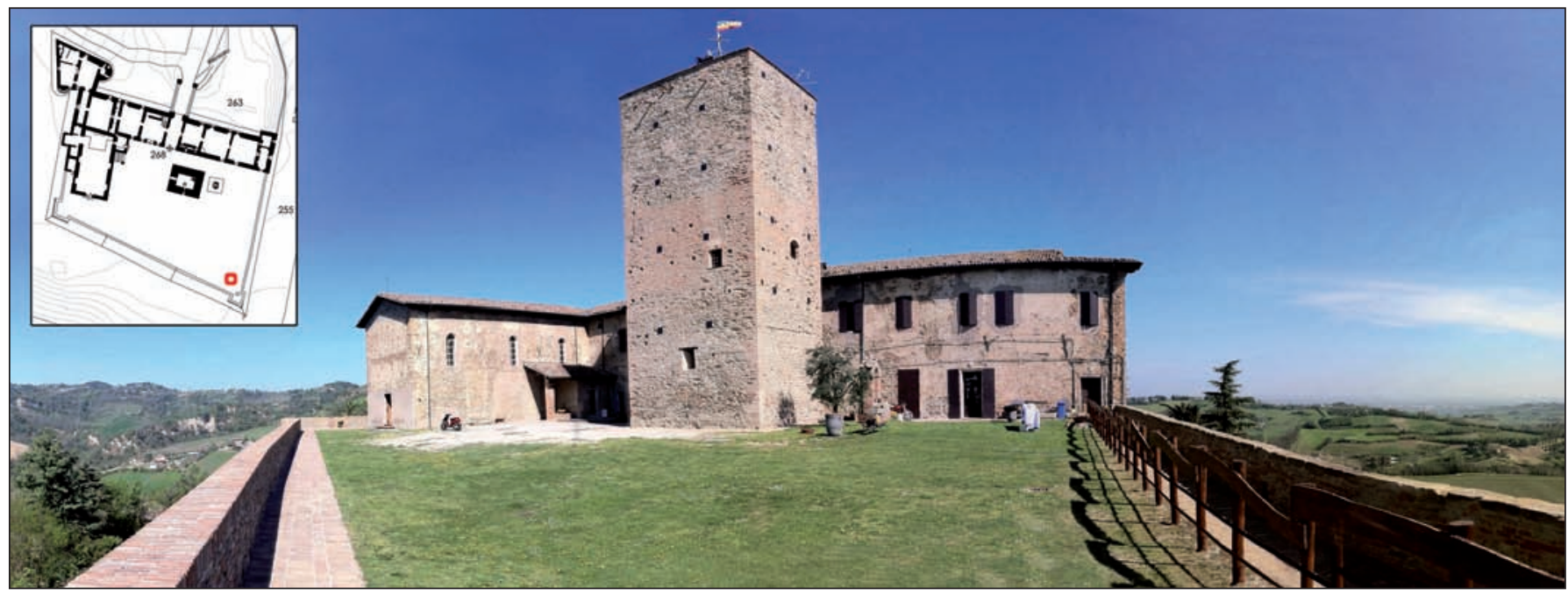

Fig. 2. Il castello di Sorrivoli (foto realizzata nel mese di aprile 2011). 

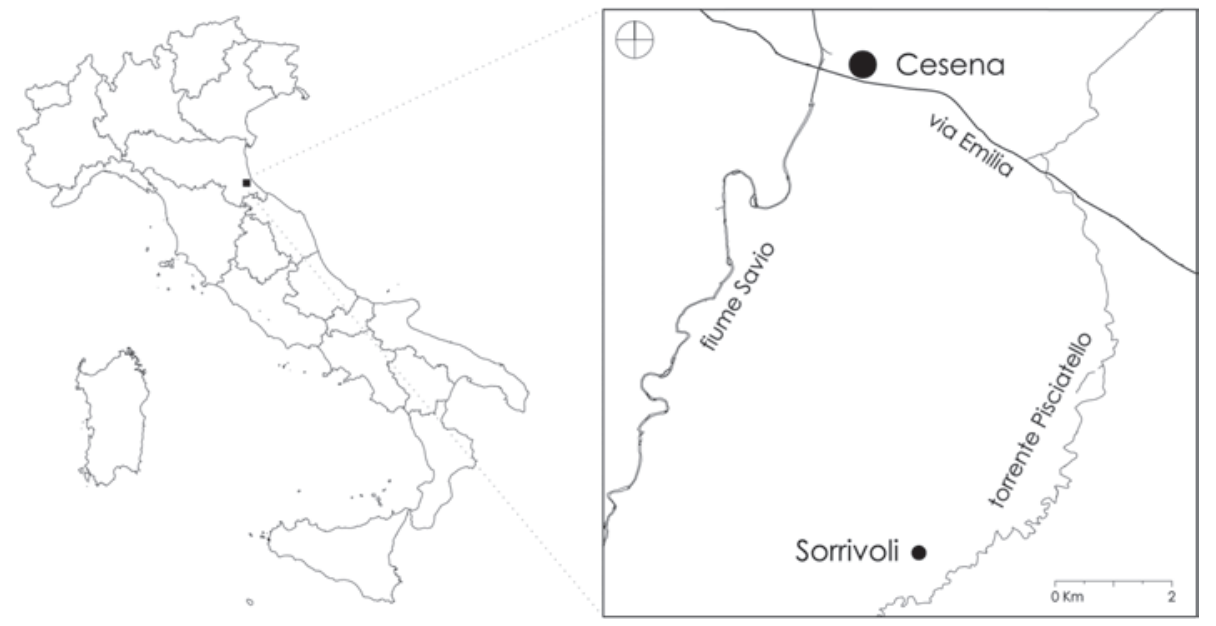

Fig. 3. Localizzazione geografica del castello di Sorrivoli.

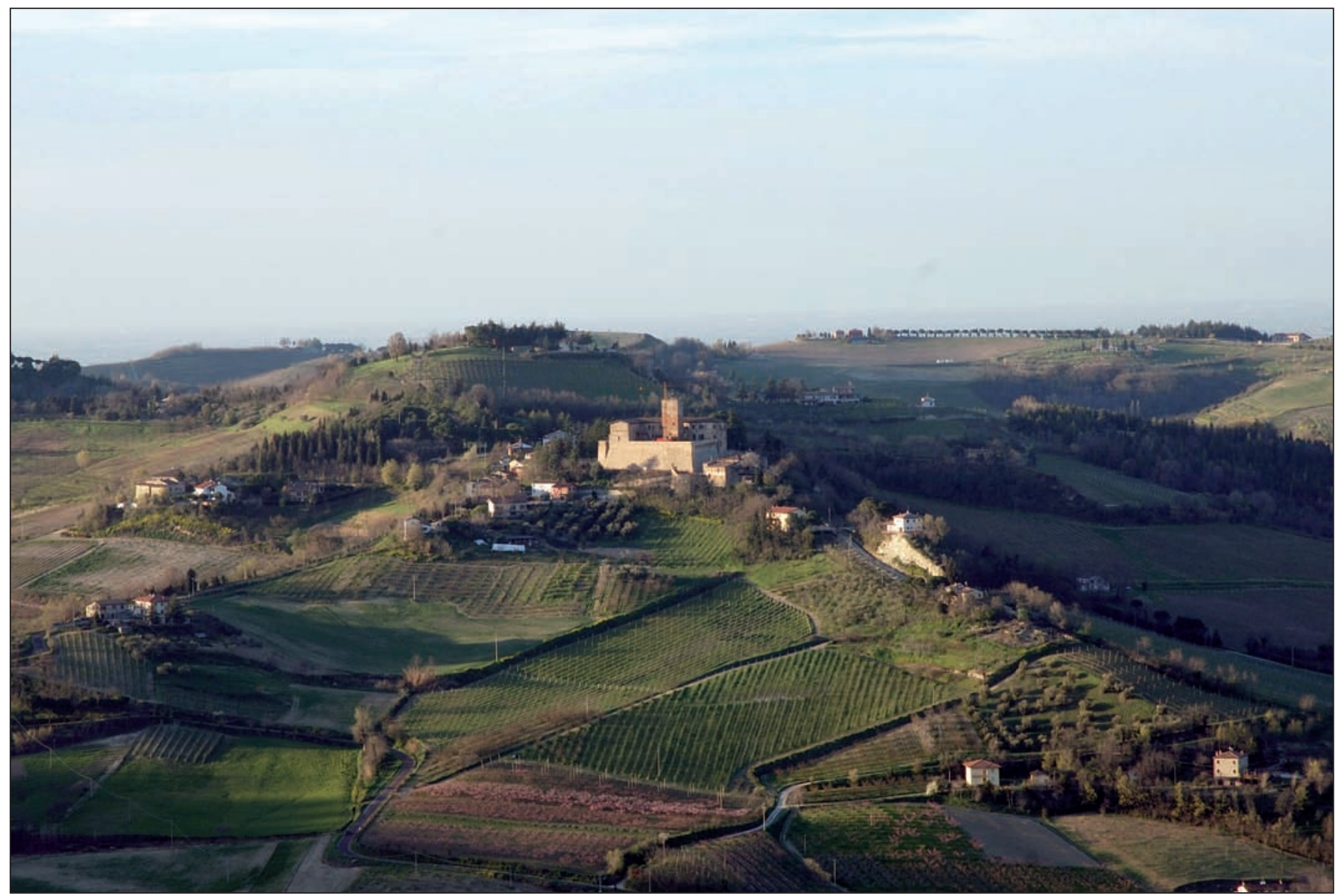

Fig. 4. Il castello di Sorrivoli visto da Sud.

L'esistenza del castrum di Sorrivoli è attestata dalle fonti scritte a partire dalla fine del $\mathrm{x}$ secolo $^{2}$. In questo

${ }^{2}$ Benericetti, 2002a, p. 149. L'Autore trascrive il testo di una pergamena datata 8 luglio 991 e conservata presso l'Archivio Arcivescovile di Ravenna con segnatura AAR F 1942. periodo l'area occupata dal complesso architettonico rientra nei possedimenti della Chiesa arcivescovile di Ravenna, la quale va consolidando il controllo su gran parte del territorio che fu dell'Esarcato grazie alle disposizioni (conferme di proprietà) contenute nei documenti imperiali e 
papali (Sassi, 2005, p. 52; Rabotti, 1993, pp. 129, 135)33. La rete di castelli allestita dagli arcivescovi serve per difendere i propri beni territoriali ma anche per impedire la crescita di signorie rurali e di autonome istituzioni comunali (Pasquali, 1996, p. 65; Sassi, 2005, p. 54).

Alla fine del XIV secolo Cesena e il suo territorio entrano nelle mire espansionistiche dei Malatesti. Nel giro di pochi anni ottengono il controllo di nuove città e castelli: Cesena (1378), il porto di Cesenatico (1382), Sorrivoli, Roversano, Teodorano (1392) e Bertinoro (1394) (Mazzatinti, 1909, pp. 70, 77; Braschi, 1738, p. 285). All'inizio del XV secolo la signoria territoriale dei Malatesti è già vastissima. Nel 1415 solo nella fascia collinare a Sud di Cesena il pontefice concede in vicariato ad Andrea Malatesta almeno 7 castelli arcivescovili (tra i quali Sorrivoli) (Fantuzzi, Monumenti, IV, p. 454; Riva, 1985, p. 277). Questo processo di ampliamento territoriale è messo in atto attraverso azioni militari, lo sviluppo di rapporti di buon vicinato e una politica di ricerca dei consensi. E' con questa intenzione, ad esempio, che $\mathrm{i}$ Malatesti di Cesena nel 1448 assecondano il desiderio della chiesa ravennate di rientrare in possesso della rocca di Sorrivoli (Fabbri, 2003, pp. 102-105). In cambio Domenico Malatesta Novello (signore di Cesena dal 1433 al 1465) riceve terreni e case a Longiano (Robertson, 1985, p. 19; Fabbri, 2003, p. 103, n. 216). Da questo momento (fino al XVIII secolo) la storia di Sorrivoli si lega con quella della famiglia Roverella. Le parti coinvolte nella permuta del 1448 sono, infatti, il procuratore di Malatesta Novello (il notaio Assalonne di ser Matteo di Giovanni) e Pietro Roverella (autorizzato dal fratello, l'arcivescovo Bartolomeo, a rappresentare la chiesa ravennate). In effetti, proprio la carriera ecclesiastica di alcuni membri di questa famiglia (Bartolomeo, Lorenzo, Niccolò e Filasio: Scardino, 2000, p. 96) permette ai Roverella di accumulare in poco tempo notevoli fortune. Nei primi anni del XVI secolo, ad esempio, la Curia di Ravenna concede la contea di Sorrivoli a Giovanni Roverella, figlio dell'arcivescovo Filasio (Rossi, 1589, p. 687; Sansovino, 1609, p. 393; Bolognesi, Lorenzini 2004, p. 68). In Romagna, nel frattempo, le principali signorie locali entrano in crisi e la Santa Sede interviene per assicurarsi il dominio diretto delle principali città (Cesena nel 1465: Vasina, 1986, pp. 178-180).

${ }^{3}$ Il 27 settembre 999, ad esempio, Ottone III rilascia all'arcivescovo Leone un diploma nel quale è sancita la giurisdizione su diversi comitati, tra i quali, quello di Cesena (Rabotti, 1993, p. 136; Vasina, 1983, p. 95).

\section{STRUMENTI E METODI DELLA RICERCA ${ }^{4}$}

Per determinare la storia costruttiva del castello si sono impiegati gli strumenti e i metodi dell'archeologia dell'architettura (Brogiolo, 1988; Doglioni, 1997; Francovich, Parenti, 1988; Parenti, 2000). Una particolare attenzione è stata posta ai seguenti aspetti dell'attività sul campo: la registrazione dei dati attraverso un ricco apparato di schede; il rilievo accurato degli elementi che costituiscono le murature (Fiorini, 2008, p. 184); il riconoscimento dei litotipi e dei siti di approvvigionamento del materiale costruttivo tramite la consulenza di specialisti del settore geologico (Zaffagnini, 2009).

In primo luogo si sono identificati e numerati i diversi corpi di fabbrica (CF) che compongono il complesso architettonico (CA). Allo stesso modo, anche i prospetti (PR) e gli elementi architettonici (EA) hanno ricevuto un numero identificativo. Il complesso architettonico è stato cos̀̀ scomposto in 10 corpi di fabbrica, 88 prospetti e 64 elementi architettonici. La posizione di queste «unità di riferimento» è stata annotata sui rilievi architettonici a disposizione (piante, sezioni e prospetti) ${ }^{5}$. Questa documentazione, unitamente all'elenco generale delle unità (scheda UR), ha favorito la comprensione del monumento e l'identificazione delle parti più interessanti sulle quali operare le successive analisi (fig. 5).

La complessa articolazione stratigrafica e le dimensioni importanti del monumento hanno suggerito una strategia di lavoro "sul campo" caratterizzata da un progressivo approfondimento delle analisi: prima di tutto si sono documentati (in pianta) i principali rapporti stratigrafici che intercorrono tra i diversi CF ( $\mathrm{i}$ «nodi stratigrafici») e successivamente si sono registrati (nei prospetti) i perimetri di tutte le US visibili nelle superfici architettoniche. Questo procedimento ha consentito una comprensione graduale e profonda del monumento.

Gli schizzi stratigrafici realizzati sul campo sono stati integrati con i dati desunti dalle osservazioni al monitor di foto (ad alta risoluzione) delle superfici architettoniche. Infine, gli strumenti per il disegno vettoriale del software AutoCAD hanno permesso di ottenere, in tempi rapidi, una versione digitale di tutta la documentazione.

In base ai dati raccolti sul campo, è stata costruita una sequenza cronologica (relativa) delle unità architettoniche più grandi $(\mathrm{CF})$. Questo ha permesso di comprendere a

\footnotetext{
${ }^{4}$ Le ricerche sono state condotte nel giugno del 2009.

${ }^{5}$ I rilievi a disposizione sono stati eseguiti nel giugno del 2000 nell'ambito di un progetto di restauro conservativo proposto in seguito al cedimento della cortina
} meridionale della rocca. 
SCHEDA UR - Unità di riferimento

Laboratorio di Archeologia dell'architettura - Università di Bologna

\begin{tabular}{|c|c|c|c|}
\hline Sito $\mathbf{n}^{\circ}$ & Sito denominazione & Comune & Località \\
\hline 12 & Castello di Sorrivoli & Roncofreddo & Sorrivoli \\
\hline
\end{tabular}

\begin{tabular}{|c|c|}
\hline CF $\mathbf{n}^{\circ}$ & $\begin{array}{c}\text { Nel } \\
\mathbf{C A} \mathbf{n}^{\circ}\end{array}$ \\
\hline 1 & $/$ \\
\hline 2 & 1 \\
\hline 3 & 1 \\
\hline 4 & 1 \\
\hline 5 & 1 \\
\hline 6 & 1 \\
\hline 7 & 1 \\
\hline 8 & 1 \\
\hline 9 & $/$ \\
\hline 10 & $/$ \\
\hline 11 & $/$ \\
\hline & \\
\hline & \\
\hline & \\
\hline & \\
\hline & \\
\hline & \\
\hline & \\
\hline & \\
\hline & \\
\hline & \\
\hline & \\
\hline & \\
\hline & \\
\hline & \\
\hline & \\
\hline & \\
\hline & \\
\hline & \\
\hline & \\
\hline & \\
\hline & \\
\hline & \\
\hline & \\
\hline & \\
\hline & \\
\hline & \\
\hline & \\
\hline
\end{tabular}

\begin{tabular}{|c|c|c|}
\hline $\mathrm{PR}^{\circ}$ & PR orient. ${ }^{1}$ & $\begin{array}{c}\mathrm{Nel} \\
\mathrm{CF}^{\circ}{ }^{\circ}\end{array}$ \\
\hline 1 & nord & 1 \\
\hline 2 & ovest & 1 \\
\hline 3 & sud & 1 \\
\hline 4 & est & 1 \\
\hline 5 & est & 2 \\
\hline 6 & est & 3 \\
\hline 7 & nord & 4 \\
\hline 8 & nord-est & 3 \\
\hline 9 & ovest & 6 \\
\hline 10 & ovest & 4 \\
\hline 11 & ovest & 6 \\
\hline 12 & ovest & 7 \\
\hline 13 & ovest & 3 \\
\hline 14 & sud & 3 \\
\hline 15 & ovest & 2 \\
\hline 16 & ovest & 8 \\
\hline 17 & nord & 8 \\
\hline 18 & est & 8 \\
\hline 19 & sud-ovest & 3 \\
\hline 21 & ovest & 1 \\
\hline 22 & nord & 1 \\
\hline 23 & est & 1 \\
\hline 24 & sud & 1 \\
\hline 25 & ovest & 10 \\
\hline 26 & sud & 10 \\
\hline 27 & sud & 9 \\
\hline 28 & est & 9 \\
\hline 29 & est & I \\
\hline 30 & est & 4 \\
\hline 31 & ovest & I \\
\hline 32 & est & I \\
\hline 33 & sud & 1 \\
\hline 34 & ovest & 1 \\
\hline 35 & nord & 1 \\
\hline 36 & est & 1 \\
\hline 37 & sud & 1 \\
\hline 38 & ovest & 1 \\
\hline 39 & nord & 1 \\
\hline 40 & est & 1 \\
\hline 41 & sud & 1 \\
\hline 42 & ovest & 1 \\
\hline 43 & nord & 1 \\
\hline 44 & est & 1 \\
\hline 45 & est & 6 \\
\hline
\end{tabular}

\begin{tabular}{|c|c|}
\hline UF $\mathbf{n}^{\circ}$ & $\begin{array}{c}\text { Dentro } \\
\text { al CF } \mathbf{n}^{\circ}\end{array}$ \\
\hline 1 & 1 \\
\hline 2 & 1 \\
\hline 3 & 1 \\
\hline 4 & 1 \\
\hline 5 & 9 \\
\hline 6 & 2 \\
\hline 7 & 1 \\
\hline 8 & 4 \\
\hline 9 & 6 \\
\hline 10 & 2 \\
\hline 11 & 1 \\
\hline 12 & 7 \\
\hline 13 & 8 \\
\hline 14 & 4 \\
\hline 15 & 5 \\
\hline 16 & 5 \\
\hline 17 & 3 \\
\hline 18 & 3 \\
\hline 19 & 3 \\
\hline 20 & 4 \\
\hline 21 & 4 \\
\hline 22 & 4 \\
\hline 23 & 4 \\
\hline 24 & 4 \\
\hline 25 & 4 \\
\hline 26 & 4 \\
\hline 27 & 4 \\
\hline 28 & 4 \\
\hline & \\
\hline & \\
\hline & \\
\hline & \\
\hline & \\
\hline & \\
\hline & \\
\hline & \\
\hline & \\
\hline & \\
\hline & \\
\hline & \\
\hline & \\
\hline & \\
\hline & \\
\hline & \\
\hline & \\
\hline & \\
\hline & \\
\hline & \\
\hline & \\
\hline
\end{tabular}

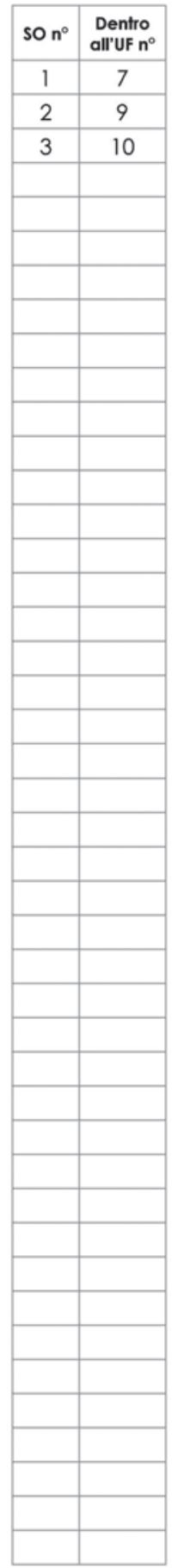

\begin{tabular}{|c|c|c|c|}
\hline EA $n^{\circ}$ & \multicolumn{2}{|c|}{ Nel PR $n^{\circ}$} & ES.TC ${ }^{2}$ \\
\hline 1 & 2 & 36 & Sì \\
\hline 2 & 3 & 33 & Sì \\
\hline 3 & 4 & 21 & Sì \\
\hline 4 & 4 & 21 & Sì \\
\hline 5 & 9 & 1 & Sì \\
\hline 6 & 15 & I & Sì \\
\hline 7 & 18 & 30 & cfr. 6 \\
\hline 8 & 14 & / & Sì \\
\hline 9 & 4 & 42 & Sì \\
\hline 10 & 4 & 21 & Sì \\
\hline 11 & 3 & 24 & Sì \\
\hline 12 & 12 & 54 & Sì \\
\hline 13 & 13 & 1 & No \\
\hline 14 & 8 & 6 & Sì \\
\hline 15 & 7 & 1 & Sì \\
\hline 16 & 6 & 51 & Sì \\
\hline 17 & 6 & I & Sì \\
\hline 18 & 6 & 51 & Sì \\
\hline 19 & 8 & 19 & Sì \\
\hline 20 & 5 & 52 & Sì \\
\hline 21 & 8 & 1 & Sì \\
\hline 22 & 9 & I & \\
\hline 23 & 9 & 1 & \\
\hline 24 & 9 & 1 & \\
\hline 25 & 9 & 49 & \\
\hline 26 & 8 & 1 & \\
\hline 27 & 18 & 53 & Sì \\
\hline 28 & 29 & 1 & Sì \\
\hline 29 & 7 & I & \\
\hline 30 & 7 & 1 & \\
\hline 31 & 17 & I & \\
\hline 32 & 29 & I & \\
\hline 33 & 31 & 32 & \\
\hline 34 & 28 & 1 & \\
\hline 35 & 30 & 1 & \\
\hline 36 & 48 & 1 & \\
\hline 37 & 55 & 1 & \\
\hline 38 & 50 & I & \\
\hline 39 & 55 & / & \\
\hline 40 & 1 & 39 & \\
\hline 41 & 35 & 1 & \\
\hline 42 & 34 & 1 & \\
\hline 43 & 56 & I & \\
\hline 44 & 2 & 1 & \\
\hline
\end{tabular}

\begin{tabular}{|c|c|c|}
\hline $\begin{array}{c}\text { Camplone } \\
\mathrm{n}^{\circ}\end{array}$ & EA $n^{\circ}$ & $\begin{array}{c}\text { Nel PR } \\
n^{\circ}\end{array}$ \\
\hline 1 & I & 7 \\
\hline 2 & I & 5 \\
\hline 3 & I & 8 \\
\hline 4 & 1 & 2 \\
\hline 5 & 1 & 2 \\
\hline 6 & / & 9 \\
\hline 7 & 5 & 9 \\
\hline 8 & 6 & 15 \\
\hline 9 & 7 & 18 \\
\hline 10 & 1 & 9 \\
\hline 11 & 1 & 18 \\
\hline 12 & I & 4 \\
\hline 13 & 14 & 6 \\
\hline 14 & 1 & 17 \\
\hline 15 & 35 & 30 \\
\hline 16 & / & 57 \\
\hline 17 & I & 58 \\
\hline 18 & I & 15 \\
\hline & & \\
\hline & & \\
\hline & & \\
\hline & & \\
\hline & & \\
\hline & & \\
\hline & & \\
\hline & & \\
\hline & & \\
\hline & & \\
\hline & & \\
\hline & & \\
\hline & & \\
\hline & & \\
\hline & & \\
\hline & & \\
\hline & & \\
\hline & & \\
\hline & & \\
\hline & & \\
\hline & & \\
\hline & & \\
\hline & & \\
\hline & & \\
\hline & & \\
\hline
\end{tabular}

1 PR orientamento: Nord, Est, Sud, Ovest, Nord-Est, Sud-Est, Sud-Ovest, Nord-Ovest. ES.TC (EA scelti come esemplificativi delle diverse tecniche costruttive): Sì, No.

Fig. 5. Scheda di registrazione delle unità architettoniche individuate nel castello di Sorrivoli. 
grandi linee le principali trasformazioni del complesso architettonico (CA). In seguito è stato allestito un «matrix» di tutte le unità stratigrafiche, in altre parole uno schema che formalizza i rapporti di cronologia relativa esistenti tra le US. I dati desunti da tutte le fonti esaminate (reperti di scavo, scritte, cartografiche, iconografiche, confronti con edifici in prossimità) hanno permesso, quindi, di attribuire le US ai diversi cantieri (fasi edilizie) e poi di ordinare in una sequenza cronologica assoluta le principali trasformazioni del castello.

All'analisi stratigrafica è seguita quella delle tecniche costruttive. Questa indagine ha permesso di confezionare un repertorio cronotipologico delle murature e degli elementi architettonici (apparati decorativi e aperture) del castello di Sorrivoli. Per quanto riguarda i criteri di registrazione dei caratteri costruttivi e il metodo di studio tipologico si è fatto riferimento a una procedura oramai collaudata che conduce alla distinzione dei manufatti sulla base delle diverse figure artigianali coinvolte nel ciclo produttivo (Bianchi, 1996; Cagnana, 1996; Mannoni, 1976; Mannoni, 1997; Mannoni, 2005; Parenti, 1987). Il lavoro sul campo è stato organizzato in tre momenti: la scelta dei manufatti rappresentativi delle diverse tipologie; la schedatura dei caratteri costruttivi e formali; il rilievo fotogrammetrico. La tecnica di rilevamento usata, la «fotomodellazione», presenta alcuni aspetti degni di nota. In primo luogo necessita esclusivamente di tre prese fotografiche (una frontale e due oblique) per ottenere, in tempi rapidi, una corretta restituzione fotografica delle superfici architettoniche (fig. 6). Non servono altri dispositivi ausiliari come scomode cornici graduate da appoggiare alla superficie architettonica per ottenere un riferimento metrico o costosi strumenti di precisione (Total Station) per il rilievo dei punti di appoggio fotografico ${ }^{6}$. Altro punto di forza della fotomodellazione risiede nell'elevata precisione

\footnotetext{
${ }^{6}$ Per campioni di m 2 di lato si applicano sul muro piccolissimi dischi di plastilina (diametro $\mathrm{cm} 1 \mathrm{ca}$.), con al centro sfere (diametro $\mathrm{mm} 0,4$ ) in plastica dura (perline per la decorazione di tessuti). La loro posizione deve corrispondere approssimativamente ai vertici di un quadrato di lato $\mathrm{m} \mathrm{2,3} \mathrm{ca}$. L'ortogonalità tra i lati non è richiesta e quindi basta una rotella metrica per eseguire questa operazione. Il lavoro prosegue con l'applicazione di altri 4 dischi all'interno del quadrato ( 2 sull'orizzontale e 2 sulla verticale). Per questa operazione si utilizza una riga graduata in alluminio munita di doppia bolla. Le distanze tra i 4 dischi sono annotate su una apposita scheda, nella quale si realizza anche un rapido schizzo di tutti i punti di controllo. Con l'ausilio di una fotocamera (corredata di certificato di calibrazione) si effettuano 3 prese fotografiche ad una distanza di $\mathrm{m}$ 3 ca.: una frontale e due oblique. Il fotopiano viene elaborato con PhotoModeler e importato nel CAD per la messa in scala, il taglio alle dimensioni prestabilite (m 2x2) e il disegno degli elementi. Con questo sistema la differenza tra la rappresentazione (il modello) e la realtà non supera i $\mathrm{mm} 3$ o 4 . La prova può essere fatta confrontando le distanze reali esistenti tra $\mathrm{i}$ dischi con quelle misurabili sul fotopiano (Fiorini, 2008, p. 184, n. 18).
}

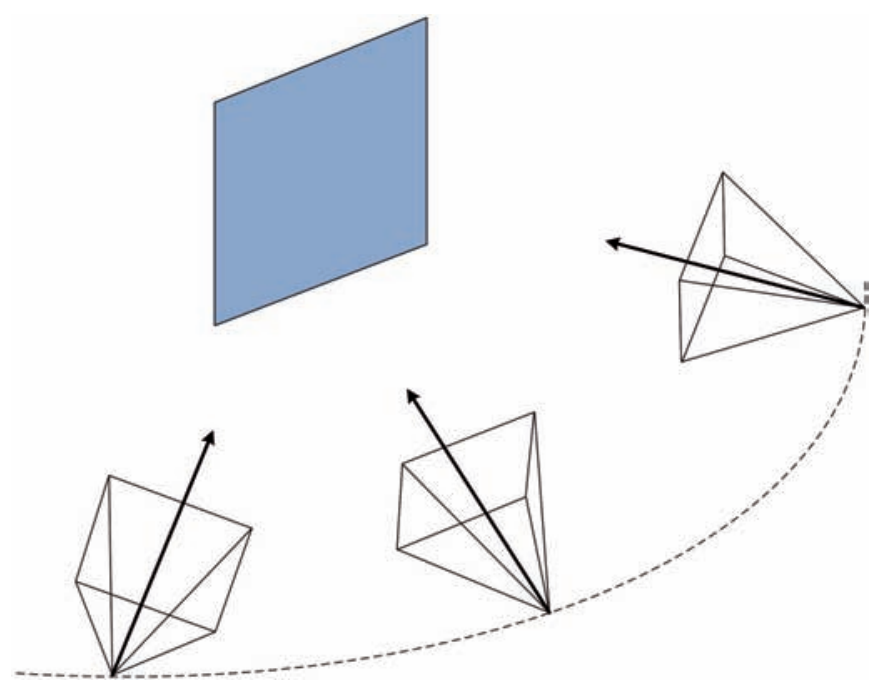

Fig. 6. Schema che illustra la posizione delle fotocamere nel rilievo fotogrammetrico di un campione di muratura.

della restituzione fotografica finale. Questa tecnica, infatti, non conduce alla realizzazione di semplici fotopiani, ma a immagini ottenute per raddrizzamento differenziale selettivo (ortofoto). In altre parole si possono definire manualmente più piani da sottoporre alla procedura di raddrizzamento (De Luca 2011; Fiorini, 2008).

Questa tecnica fotogrammetrica inoltre viene impiegata, già da qualche anno, per ottenere la restituzione tridimensionale degli elementi strutturali e della stratigrafia muraria (fig. 7) (Archetti, Fiorini, c.s.). La struttura architettonica è difatti un volume tridimensionale e i caratteri che vengono individuati nel corso delle indagini archeologiche (informazioni stratigrafiche, metriche e formali) si distribuiscono nelle tre dimensioni (Brogiolo, 2002, p. 23). Adottare pertanto i soli supporti grafici bidimensionali, ad esempio il diffusissimo fotopiano, significa perdere informazioni sulla componente tridimensionale delle evidenze stratigrafiche (Doglioni, 1997, p. 142). Tornando al caso di Sorrivoli, in totale si sono eseguiti 18 rilievi fotogrammetrici: 13 paramenti murari e 5 elementi architettonici. Per ogni campione di muratura si sono misurate, al millimetro, le dimensioni relative allo spessore, alla lunghezza e alla larghezza di ogni elemento. Le misurazioni sono state operate al computer ad eccezione dei paramenti in laterizi per i quali si è preferito il rilievo diretto tramite riga metallica graduata. Le misurazioni hanno interessato esclusivamente i materiali che conservavano integre le dimensioni originarie e non si sono pertanto considerati i mattoni spezzati o quelli il cui stato di conservazione era tale da non poter identificare con sicurezza i bordi originari. Per ogni campione di muratura 
Fig. 7. La restituzione tridimensionale degli elementi strutturali e della stratigrafia muraria: in alto la chiesa torre di Monte Erno (Galeata - FC). Elaborato da Archetti, Fiorini, c.s. di San Bartolomeo Apostolo (Cesena - FC); in basso la
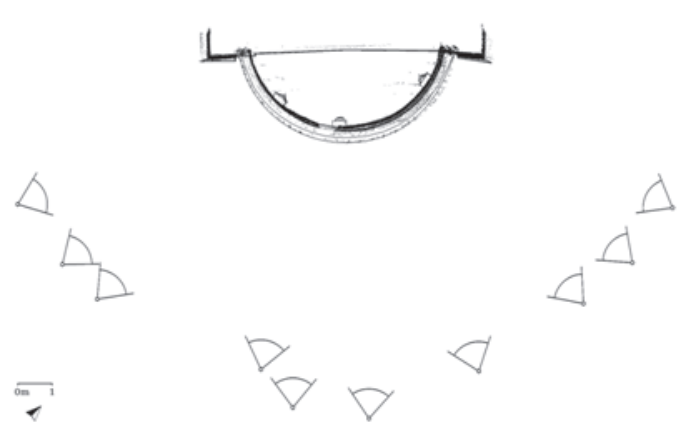

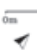
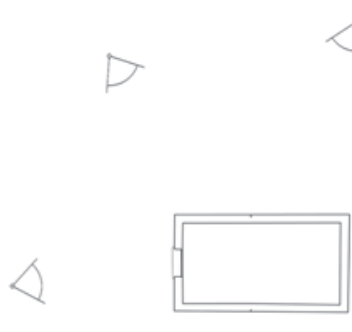

$+$

si sono calcolate la media e la moda (valore più ricorrente) di ogni dimensione (spessore, lunghezza e larghezza). In primo luogo, comparando le dimensioni di tutti i laterizi esaminati si è potuto accertare l'appartenenza allo stesso cantiere di alcuni corpi di fabbrica e di accertare l'estraneità di diversi interventi di restauro al progetto originario. In secondo luogo, è stato possibile avanzare ipotesi circa la datazione di alcune strutture murarie in base delle corrispondenze dimensionali riscontrate nei laterizi di alcuni edifici di cronologia nota posti nella città di Cesena.

Lo studio archeologico dei castelli medievali non può essere disgiunto da un approfondimento di tipo geologico, riguardante i caratteri litologici delle pietre che affiorano in prossimità degli insediamenti e di quelle impiegate nel monumento in esame. Questi dati si possono utilizzare per arricchire le conoscenze su determinati aspetti storici come ad esempio la "possibilità di spesa" dei committenti. Nel caso specifico di Sorrivoli, sono stati individuati i bacini di approvvigionamento del materiale costruttivo e $\mathrm{i}$ rapporti tra $\mathrm{i}$ caratteri litologici degli affioramenti rocciosi e la lavorazione adottata per ottenere gli elementi da costruzione.
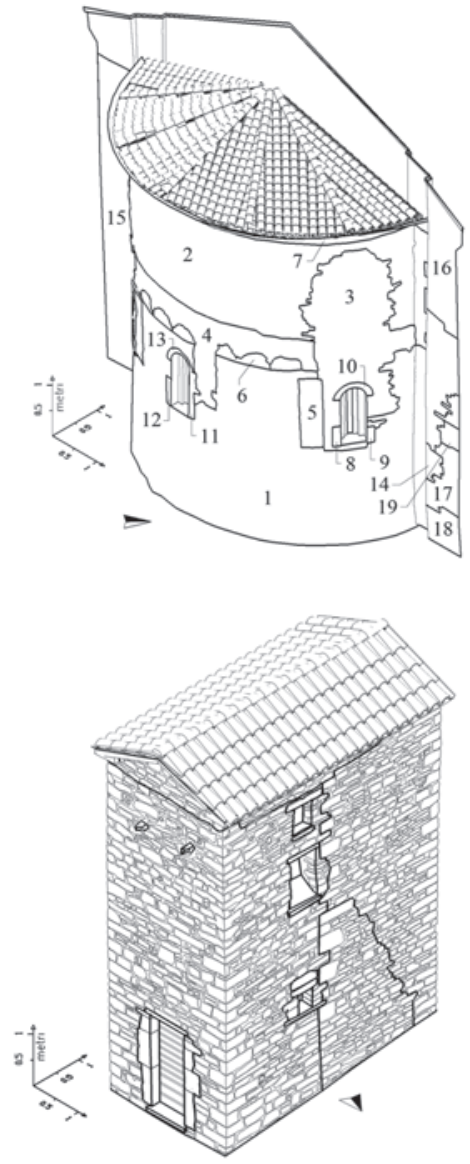

In genere, i castelli della Romagna sono costruiti con pietra locale. Spesso si utilizzano più siti di approvvigionamento e questo determina una sostanziale disomogeneità litologica dei paramenti. La roccia scelta per le lavorazioni raffinate (es. la riquadratura) è spesso massiva (priva di suddivisioni laminari). La roccia non lavorata (o al massimo spaccata) è invece nella maggior parte dei casi laminata (una caratteristica che favorisce la tecnica «a spacco»). Tutti gli elementi delle strutture militari (torri e cinte murarie) sono sempre ottenuti, a prescindere dal tipo di lavorazione, da rocce con grado di cementazione media o forte (vale a dire compatte) (Fiorini, 2010b).

La qualità dei rilievi pregressi ha permesso di operare una serie di analisi di tipo geometrico-dimensionale finalizzate all'individuazione dell'unità di misura premetrica utilizzata per la progettazione del castello ${ }^{7}$. In genere, questo tipo di analisi può fornire indicazioni sulla cronologia del manufatto e sulle maestranze coinvolte nel cantiere

\footnotetext{
${ }^{7}$ Sull'analisi metrologica dell'edilizia storica v.: Docci, Maestri, 1984, pp. 171174; Docci, Maestri, 1992, pp. 155-166; Fiorani 2004; Ghiretti, Ottoni, 2009; Luschi, 2006; Salvatori, 2006; Zupko, 1997.
} 
edilizio. Leggere il rilievo usando le unità di misura in uso all'epoca della costruzione permette, inoltre, di mettere in evidenza variazioni dimensionali e irregolarità formali che possono aiutare a comprendere la presenza di fasi costruttive differenti. L'unità di misura impiegata nel dimensionamento degli elementi costruttivi (dimensioni al rustico di porte e finestre, altezza di quattro o cinque filari di mattoni e dei relativi giunti di malta, etc.) può, infatti, essere peculiare di un'area culturale ben delimitata nel tempo e nello spazio. Il problema principale nell'applicazione dell'analisi metrologica consiste nella carenza di dati precisi in merito all'entrata in vigore e alla durata dei sistemi di misura. Per tale ragione la datazione di un manufatto architettonico non si può basare esclusivamente sui risultati dell'analisi metrologica. La metrologia antica può servire invece per confermare o smentire le indicazioni cronologiche ottenute con altri metodi.

Per rendere più efficace la comunicazione delle interpretazioni archeologiche - a corredo dei rilievi tradizionali (piante, sezioni e prospetti), che risultano in alcune zone poco descrittivi della struttura architettonica - è stato elaborato un modello schematico della struttura architettonica con sovrapposizione dei rilievi stratigrafici. Il programma impiegato (SketchUp, della @Last) ha facilitato l'intero processo di disegno tridimensionale grazie ai comandi di «modellazione speditiva». Inoltre, è stato possibile ottenere una distinta caratterizzazione grafica delle US per appartenenza alla fase costruttiva. Il «modello stratigrafico tridimensionale» è stato impiegato infine per ricostruire graficamente la successione degli assetti architettonici che l'edificio ha avuto nella sua storia. In primo luogo, si sono visualizzate le US appartenenti alla fase edilizia d'interesse. Dopodiché si è proceduto con il completamento delle forme architettoniche originali in base ai dati desunti da tutte le fonti esaminate.

\section{DESCRIZIONE DELLO STATO ATTUALE}

La rocca presenta una pianta quadrangolare con superficie d'ingombro di circa 2500 metri quadri e fronte d'ingresso rivolto a Nord. L'angolo Nord-Ovest è occupato da una torre a pianta quadrangolare con cantonali in laterizio e paramenti in elementi litici non lavorati o al massimo spaccati (fig. 8, CF2).

Questo edificio è inglobato nelle strutture di un bastione (CF3) appuntito in direzione della strada principale che conduce al castello. I paramenti esterni dell'imponente corpo di fabbrica sono realizzati con laterizi mentre quelli interni sono costituiti da elementi litici. La forma della sua pianta è altrettanto particolare: «ad asso di picche». Le facce, lunghe ben 19 metri, sono rettilinee e i fianchi sdoppiati: una parte arrotondata e sporgente (orecchione), l'altra rettilinea e arretrata. L'edificio raggiunge un'altezza massima di circa 15 metri. Interessanti i suoi elementi architettonici. Il confine tra la scarpa basamentale e il paramento verticale è marcato da un cordolo di medio spessore $(\mathrm{cm} \mathrm{24})$ costituito da laterizi sagomati a semicerchio convesso. Si segnala, inoltre, la presenza di bombardiere «alla francese», in altre parole, ampie feritoie per armi da fuoco, caratterizzate da un vano a clessidra e da una fenditura rettilinea (orizzontale).

Il fronte meridionale e quello orientale della rocca sono protetti da altre tre torri angolari in materiali misti: litici e laterizi (CF8-10). La corte interna è oggi delimitata da due lunghi edifici: a Ovest l'attuale chiesa (CF6) e a Nord un corpo di fabbrica, su tre livelli, oggi destinato a soddisfare funzioni abitative e ricreative (CF4).

In posizione decentrata si trova il mastio (CF1) a pianta quadrangolare $(\mathrm{m} 6,8 \times 8,2)$, con superficie d'ingombro di circa 56 metri quadri. Questa torre presenta uno sviluppo in elevato su quattro piani (sormontati da una terrazza) e raggiunge un'altezza massima di circa 18 metri. Al disotto del piano terra si trova una cisterna a pianta quadrangolare, con superficie d'ingombro di circa 5 metri quadri. Le murature della torre sono costruite con mattoni e pietra non lavorata o al massimo spaccata.

La cartografia storica informa sull'esistenza di un terzo edificio che delimitava, fino alla metà del XIX secolo, il lato Sud della corte (CF5). Il fronte Est della rocca è, invece, caratterizzato dalla presenza di un passaggio pedonale sopraelevato (CF7), largo circa 70 centimetri, in materiali misti (litici e laterizi), ora in disuso. Sopra l'apertura si riconosce un lungo vano che ospitava, probabilmente, la trave lignea («bolzone») utilizzata per il sollevamento del ponticello levatoio.

In prossimità del complesso architettonico sono osservabili i ruderi di due edifici: una struttura a pianta circolare (CF12), interpretabile come torrione della cinta esterna del castello; una chiesa (CF11), demolita dopo la seconda guerra mondiale, il cui fronte d'ingresso è osservabile in una fotografia realizzata prima del 1944. Questo edificio fu danneggiato dai bombardamenti durante la seconda guerra mondiale e pertanto si decise di aprire al culto l'ala Ovest (CF6) del castello (Bolognesi, Lorenzini, 2004, pp. 81, 83, 85-87).

Della cinta muraria in elementi misti (litici e laterizi) che difendeva la rocca di Sorrivoli sono individuabili solo brevi tratti nel lato Est della collina, là dove oggi si trova una lunga fila di abitazioni private. 


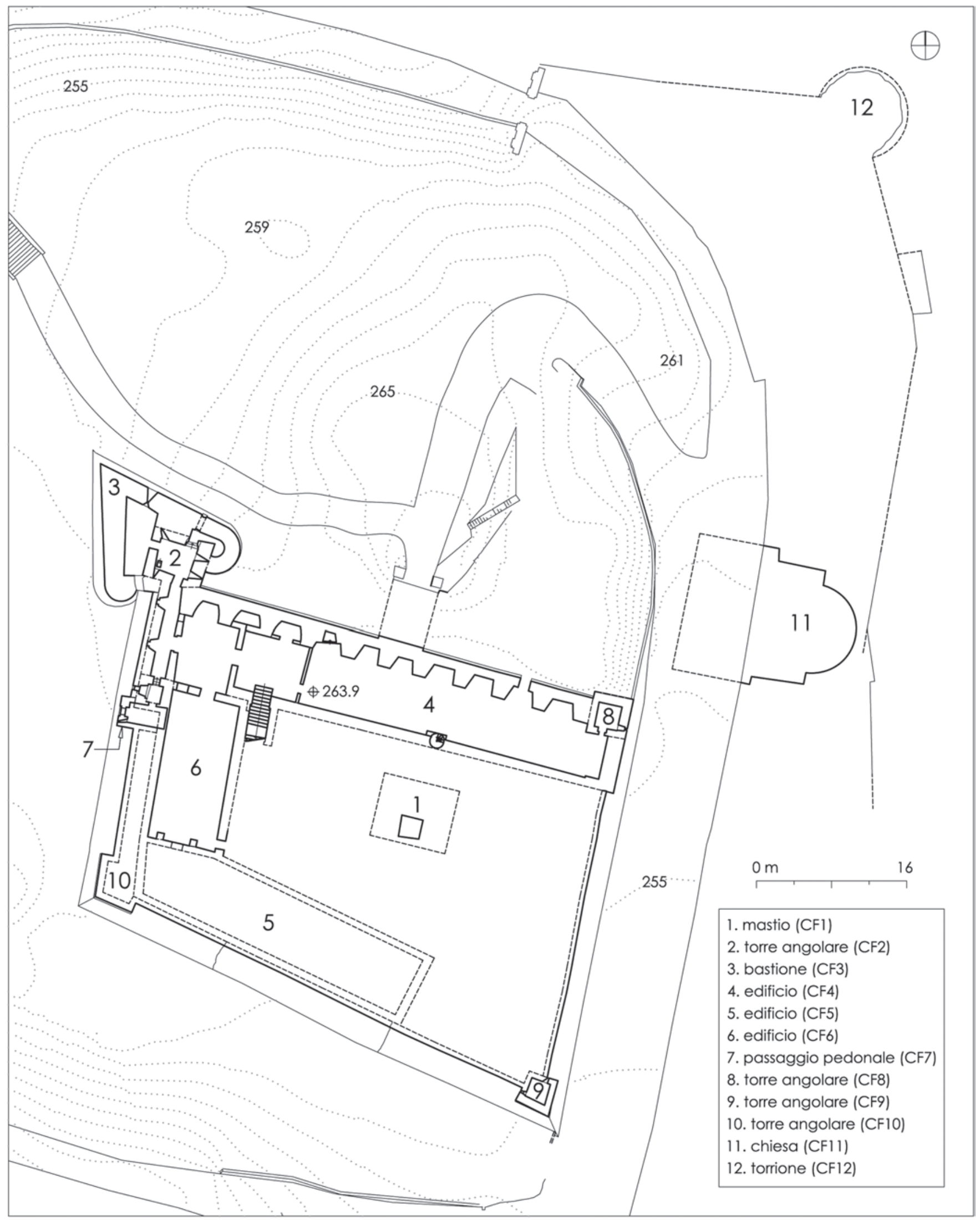

Fig. 8. Planimetria del castello di Sorrivoli con individuazione dei diversi corpi di fabbrica. Elaborazione dei rilievi eseguiti nell'ambito di un progetto di restauro conservativo proposto in seguito al cedimento della cortina meridionale della rocca (progettisti: dott.Ing. Marco Ceredi; dott.Arch. Giulia Gualterotti. Elaborazione grafica geom. Gabriele Fratti). 


\section{FASI EDILIZIE E SINTESI STORICO-ARCHEOLOGICA}

Il castrum Suripule è menzionato nei manoscritti a partire dal X secolo (Benericetti, 2002a, p. 149). In questo periodo il complesso architettonico appartiene alla rete di castelli allestita dagli arcivescovi di Ravenna per difendere i propri beni territoriali e per impedire la crescita di signorie rurali e di autonome istituzioni comunali. Nel 991 lo spazio edificabile all'interno del castello non è ancora esaurito e alcuni uomini chiedono all'arcivescovo il permesso di costruire nuove abitazioni. In cambio s'impegnano a custodire, vigilare e difendere il castello, il quale è protetto, probabilmente, da strutture piuttosto semplici in materiale deperibile, come terrapieni e palizzate lignee. È questo, ad esempio, il tipo di sistema difensivo che gli scavi archeologici hanno riportato alla luce nel castello di Rontana (Brisighella - RA) e in molti altre fortificazioni dell'Italia centro-settentrionale (Cirelli, 2009, p. 347).

L'analisi stratigrafica dell'elevato - integrata dall'esame delle fonti documentarie e da tutte le altre indagini di approfondimento (tipologiche, geologiche, metrologiche, mensiocronologiche e comparative) - ha, però, dimostrato che gli edifici più antichi, oggi conservati in elevato, rispondono a modelli trecenteschi. In totale, si sono riconosciute 7 fasi edilizie che definiscono i momenti salienti dello sviluppo architettonico del castello di Sorrivoli a partire dagli ultimi decenni del XIV secolo fino ai primi anni del XXI secolo (figg. 9-10). In questa sede si descrivono le fasi edilizie più significative (I-IV).

\section{Fase I (ultimi decenni del xIV secolo)}

Appartengono a questa fase il mastio (CF1) e i vicini edifici a pianta rettangolare (CF5 e CF6) posti nell'area sommitale del castello. Il materiale litico impiegato per realizzare le murature di questi edifici non proviene dagli affioramenti locali (rocce con un basso grado di cementazione che mal si adattano all'impiego negli elevati) ma da bacini di approvvigionamento distanti da Sorrivoli almeno $3 \mathrm{~km}$ (fig. 11: campione 5, tipo murario B2, litotipo 3). Si tratta, prevalentemente, di arenaria non lavorata o al massimo spaccata, di color grigio con sfumature violacee, caratterizzata dalla presenza di laminazione e di forte cementazione. Questi elementi litici sono combinati con mattoni prevalentemente interi (fig. 12: tipi murari B2 e B4). Nei castelli della Romagna le murature di questo tipo sono documentate a partire dal XIII secolo e soprattutto nel XIV (Fiorini, 2010b). Nel Medioevo, inoltre, l'uso del mattone intero come materiale da costruzione rimanda a una prassi fortificatoria diffusa in particolare nel XV secolo

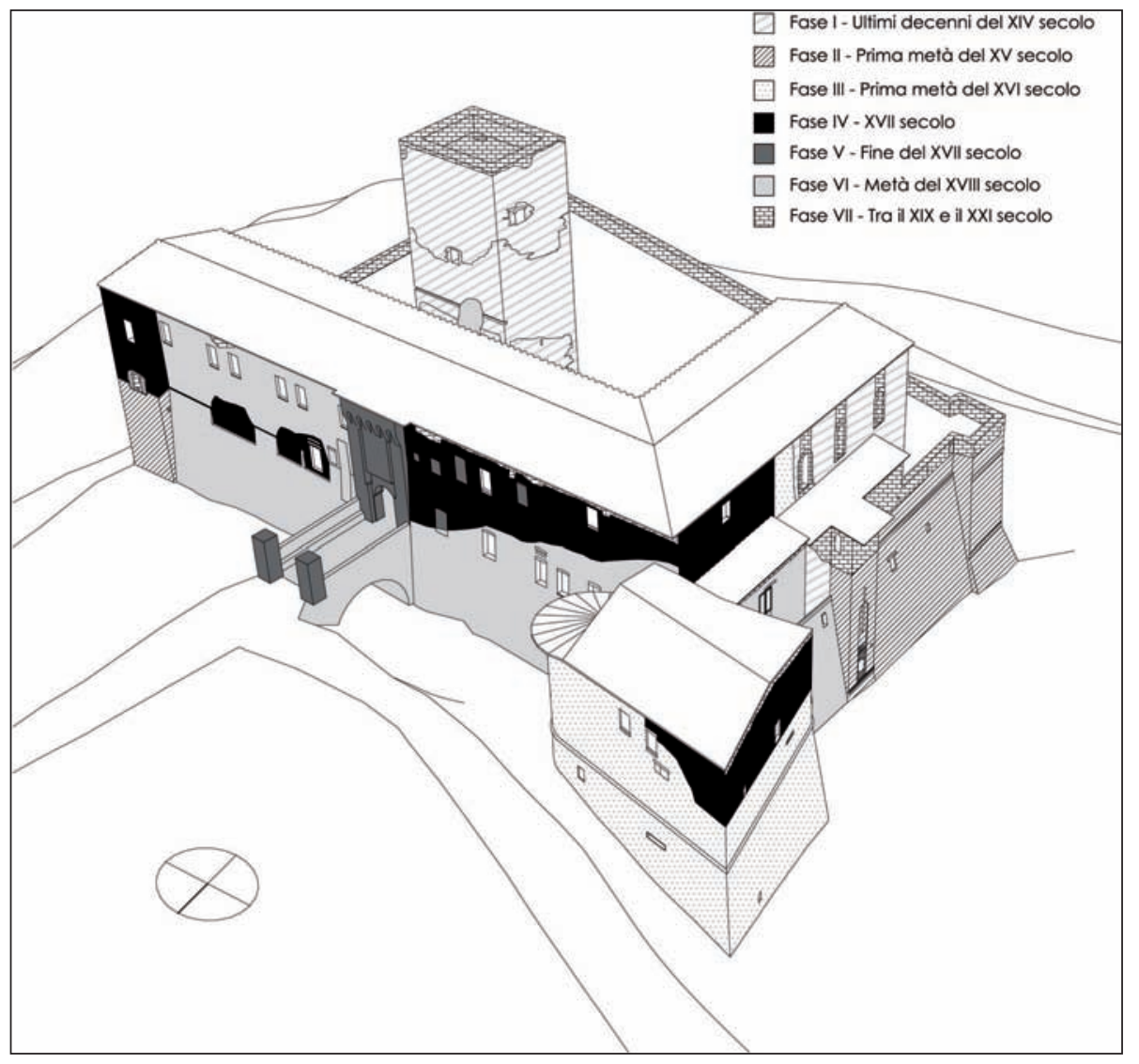

Fig. 9. Modello tridimensionale della rocca di Sorrivoli (Roncofreddo, FC) con individuazione delle diverse fasi edilizie (elaborazione grafica Emanuela Maroni, Alberto Urcia). 
FASE VII

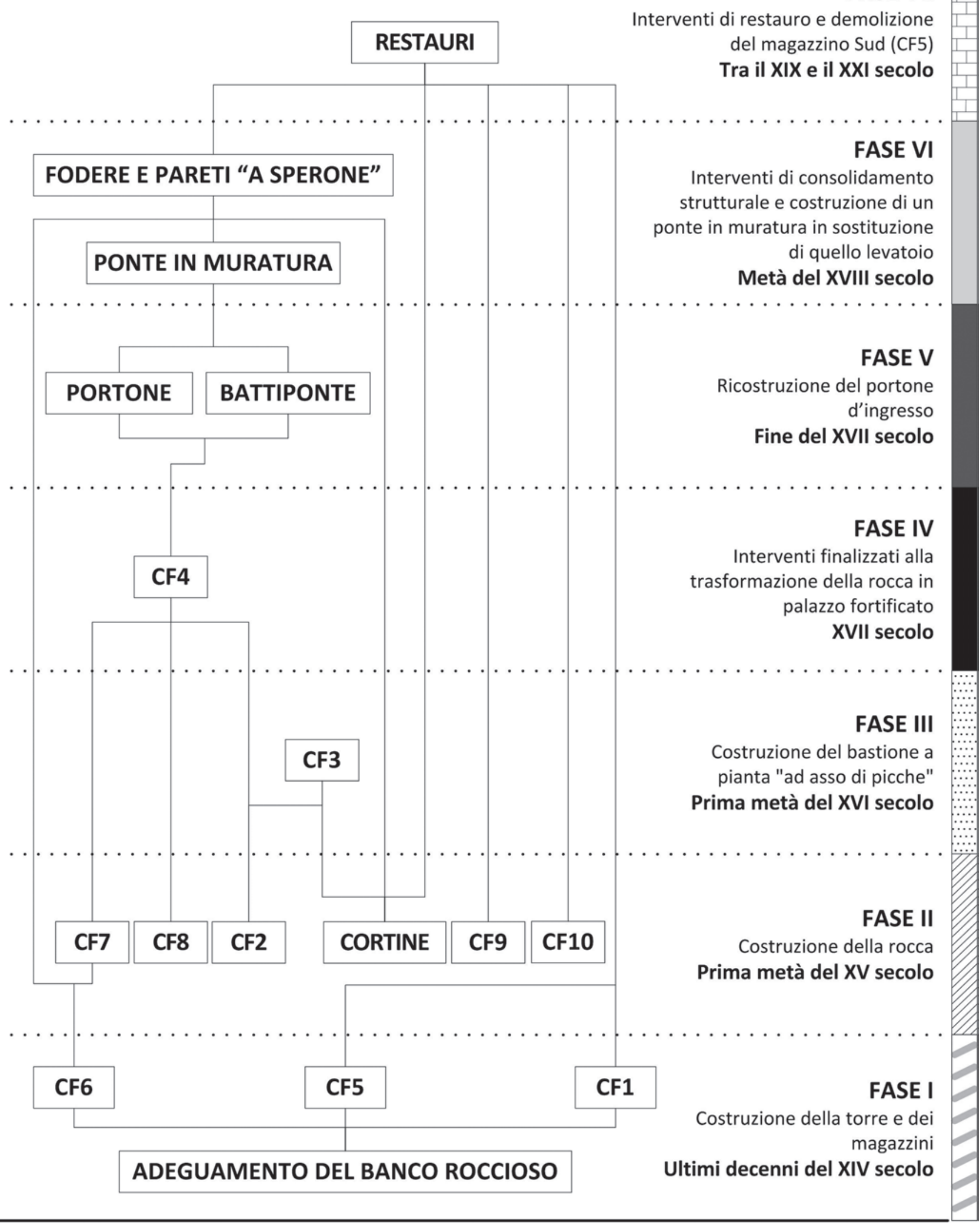

Fig. 10. Diagramma stratigrafico del castello di Sorrivoli, organizzato per corpi di fabbrica, con indicazione delle diverse fasi edilizie e relativi inquadramenti cronologici. 


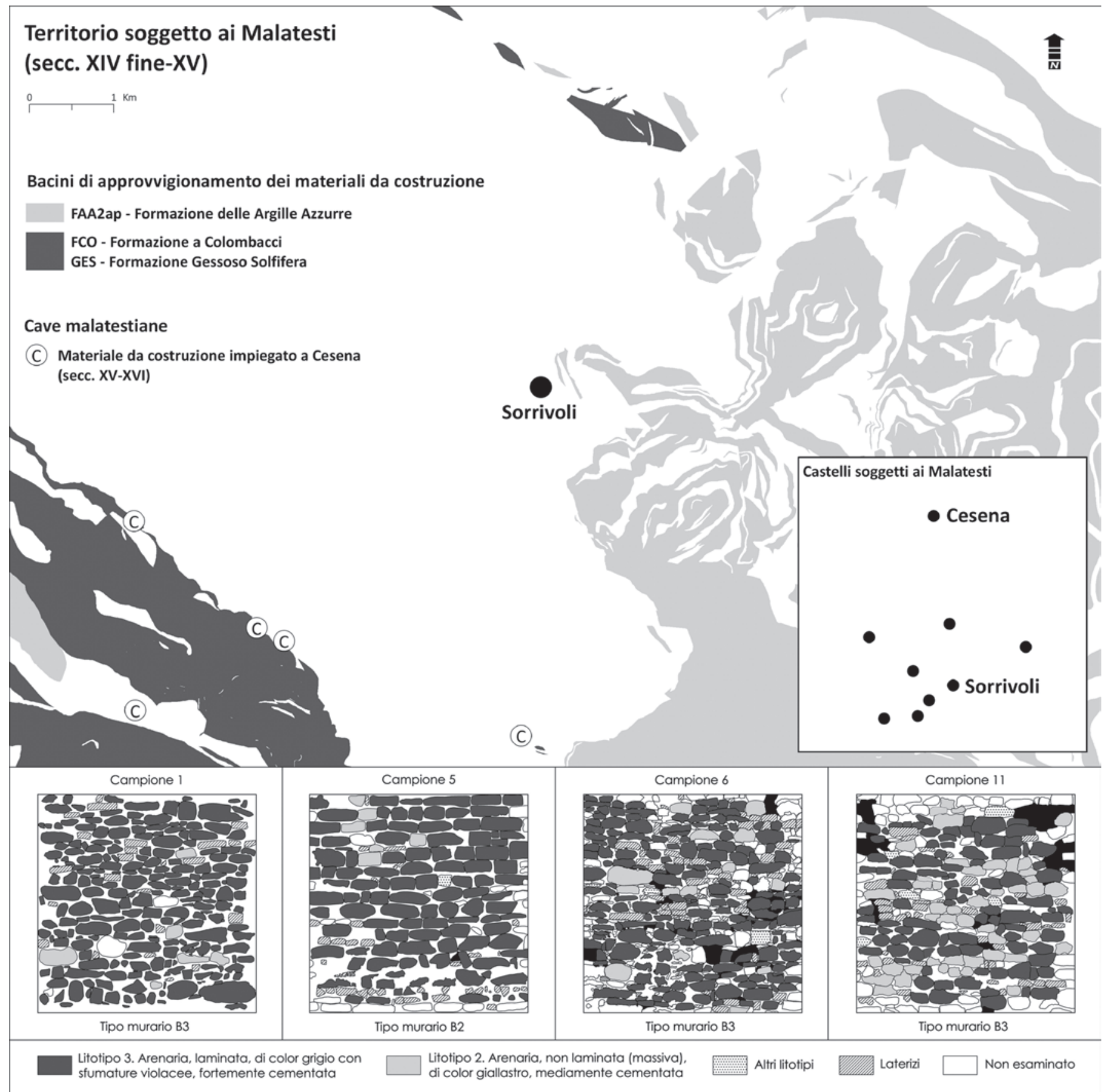

Fig. 11. Sorrivoli e i siti medievali di approvvigionamento del materiale costruttivo (elaborato da Augenti et alii, 2010, p. 82, fig. 12; indagini geologiche a cura di Fabio Zaffagnini). Nella carta sono individuate anche le cave malatestiane sfruttate per la costruzione di alcuni edifici di Cesena (Veggiani, 1968, pp. 343-367).

(Fiorini, 2010a, p. 82, fig. 13; Perogalli, 1999, p. 65). Allo stesso periodo sembrano indirizzare anche le analisi metrologiche condotte sulle strutture del mastio (CF1): l'unità di misura utilizzata per progettare l'edificio è il braccio quattrocentesco in uso a Cesena e nei suoi territori al tempo della dominazione malatestiana $(\mathrm{cm} \mathrm{55,8).} \mathrm{Così,} \mathrm{ad}$ esempio, lo spessore murario al piano terra corrisponde a circa 3 braccia (m 1,72), mentre l'ingombro della pianta (diagonale Nord-Est) equivale a circa 19 braccia (m 10,62) (fig. 13). L'altezza della torre, inoltre, corrisponde perfettamente a 33,5 braccia $(m$ 18,11) (fig. 14: A) .

\footnotetext{
${ }^{8}$ L'equivalenza in centimetri del braccio è ricavata dal rilievo diretto di una lapide ( $\mathrm{cm} 180$ x $55 \mathrm{ca}$.) conservata nella biblioteca Malatestiana di Cesena e proveniente dalle logge del palazzo comunale. Nel manufatto sono scolpite le sagome dei materiali costruttivi e le misure lineari regolamentate dal comune nel XV secolo (Montalti, 1986, p. 112, fig. 101; Pasini, 2002, pp. 129-130).
} 


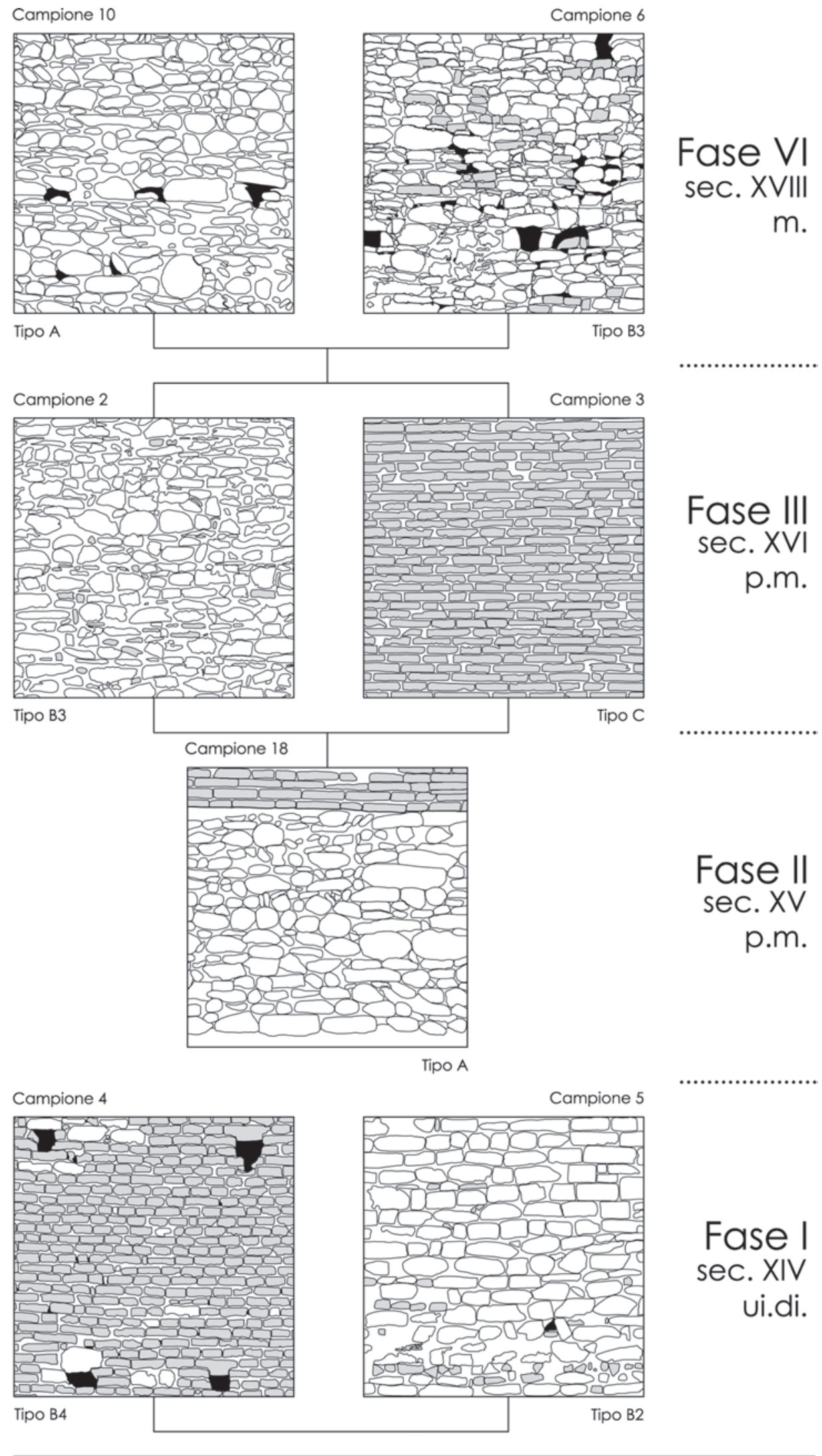

Fig. 12. Le tipologie murarie individuate nel castello di Sorrivoli con indicazione delle diverse fasi edilizie e relativi inquadramenti cronologici: I - ultimi decenni del XIV secolo; II - prima metà del XV secolo; III - prima metà del XVI secolo; VI - metà del XVIII secolo (indagini a cura di Andrea Fiorini ed Emanuela Maroni). 

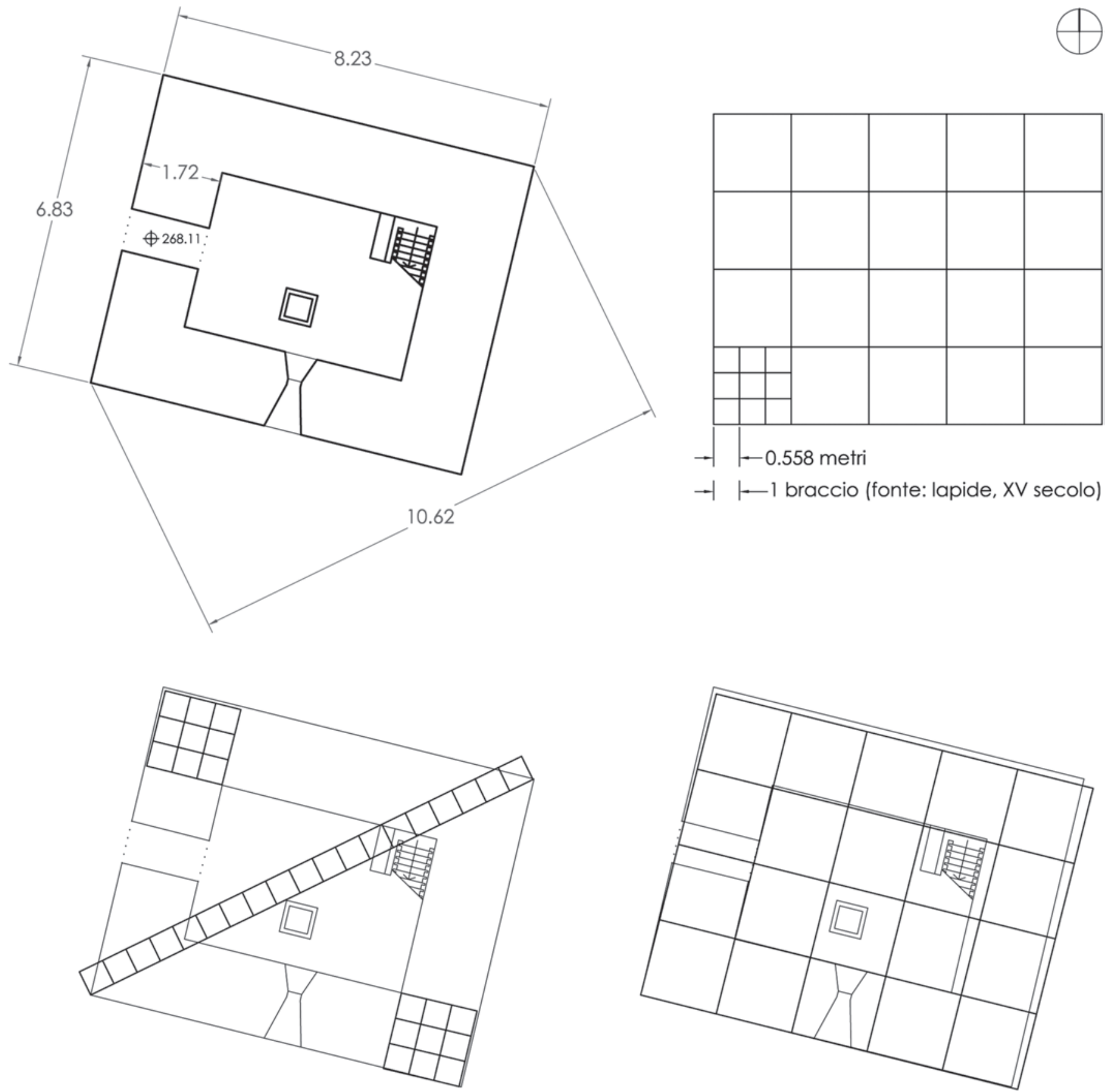

$0 \mathrm{~m}$ 2

Fig. 13. Analisi metrologica condotta sulla pianta del mastio (indagini a cura di A. Fiorini). Elaborazione dei rilievi eseguiti nell'ambito di un progetto di restauro conservativo proposto in seguito al cedimento della cortina meridionale della rocca (progettisti: dott.Ing. Marco Ceredi; dott.Arch. Giulia Gualterotti. Elaborazione grafica geom. Gabriele Fratti).

Le finestre di questo edificio sono tutte archivoltate con estradosso decorato da una fila di mattoni disposti «in chiave» (una tipologia diffusa negli edifici tardomedievali). La porta-finestra, inoltre, trova un confronto stringente con quella della torre Vanga di Trento (metà del XIV secolo: Giovannini, Parenti, 2006, p. 75). È certo, infine, che il mastio doveva già esistere nel ' 300 poiché all'interno di un vano (probabilmente una latrina) furono gettati, a partire da quel periodo (e fino al secolo successivo), recipienti ceramici non più utilizzabili9.

9 Il materiale ceramico venne recuperato tramite uno scavo «non controllato» nel 1981 (Gelichi, 1997, pp. 83-100). 


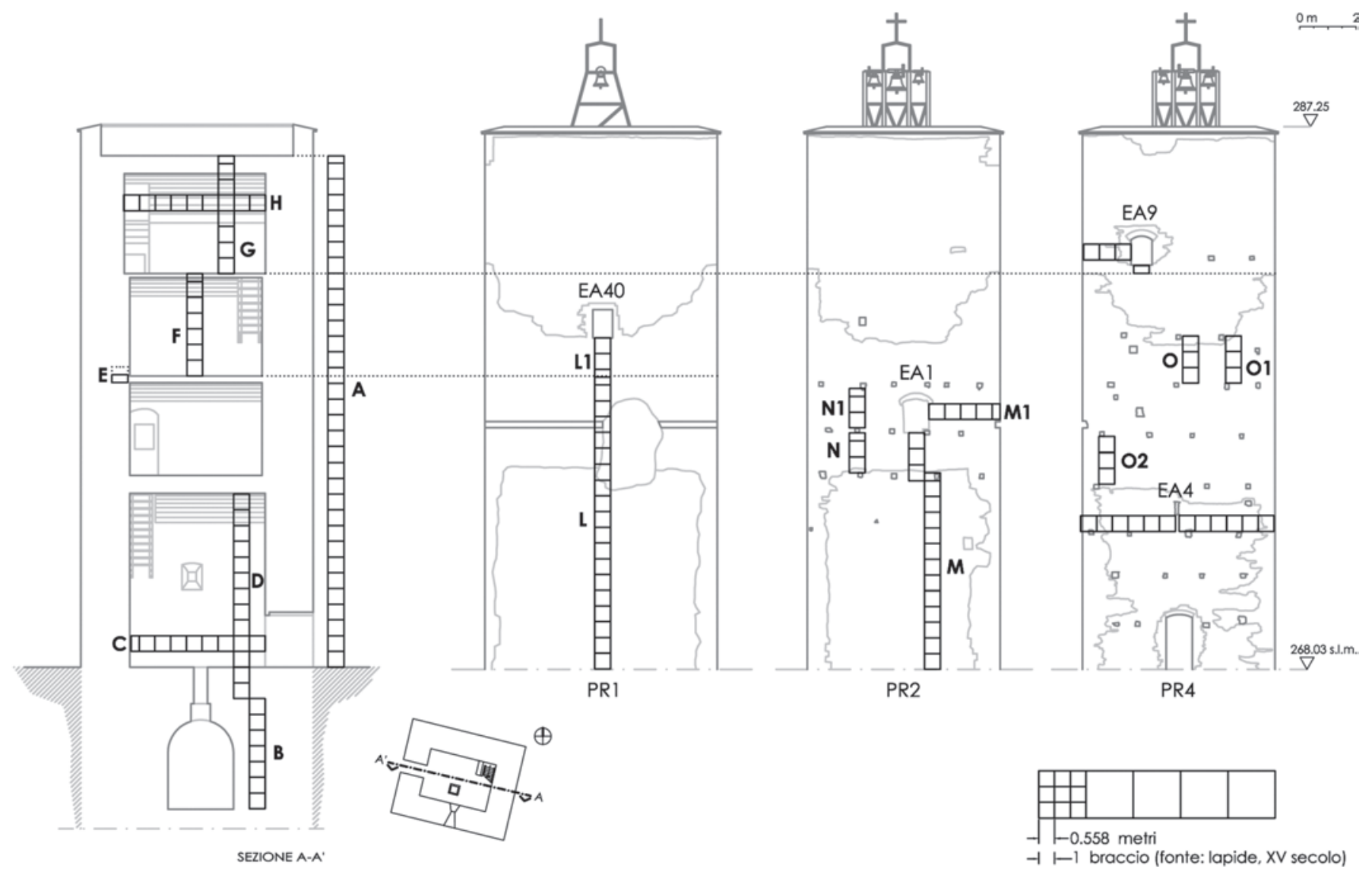

Fig. 14. Analisi metrologica condotta sui prospetti del mastio (indagini a cura di V. Archetti). Elaborazione dei rilievi eseguiti nell'ambito di un progetto di restauro conservativo proposto in seguito al cedimento della cortina meridionale della rocca (progettisti: dott.Ing. Marco Ceredi; dott.Arch. Giulia Gualterotti. Elaborazione grafica geom. Gabriele Fratti).

Nel XIV secolo la costruzione di torri in Romagna era un'impresa edilizia tutt'altro che rara. La torre di Monte Battaglia (Casola Valsenio - RA), ad esempio, viene costruita nell'ultimo decennio del XIV secolo, periodo nel quale il castello entra a far parte dei possedimenti della famiglia Alidosi (Fiorini, 2009, p. 343). Sempre nel Trecento, per volere dei Guidi, viene innalzata la torre di Dovadola $(\mathrm{FC})^{10}$.

\footnotetext{
${ }^{10}$ La datazione della torre di Dovadola viene suggerita dalle seguenti caratteristiche architettoniche. Nel prospetto Sud-Ovest del mastio è presente una portafinestra rettangolare, ben ammorsata al paramento, munita di mensole, con profilo a guscio, sormontate da un architrave, riquadrato e stipiti compositi, in elementi lavorati allo stesso modo. L'apertura è conclusa da un arco di scarico, a sesto acuto, costituito da 15 elementi, sagomati a forma di cuneo dentato, con chiave a risalto. Nella lunetta è incisa la data '"M'»CCC'” 'XXXX VIIII'” sormontata da due leoni rampanti, oggi quasi irriconoscibili. Questa portafinestra appare nel complesso piuttosto singolare. I cunei dentati caratterizzano l'architettura toscana del XII e XIII secolo (De Maio, 2007, p. 266), mentre le mensole sono simili a quelle presenti nelle aperture degli edifici quattrocenteschi dell'Appennino bolognese (Bertacci et alii, 1972, pp. 62.F, 65.F), della Lunigiana (Ferrando Cabona, Crusi, 1981, p. 184, D 2a; Ferrando Cabona, Crusi, 1988, p. 163, D/2) e dell'alta Val di Nievole (Quiros Castillo, 1992, p. 733, tav. 2, A2). La porta-finestra potrebbe, dunque, collocarsi a metà strada tra due diverse soluzioni architettoniche. In effetti, la data incisa nella lunetta rimanda al XIV secolo (1339).
}

Ai Manfredi, infine, si attribuisce la realizzazione della torre a pianta circolare (con sperone «a becco») presente nel $c a$ strum di Ceparano (Brisighella - RA) ${ }^{11}$.

${ }^{11}$ Il castro Cipariano è menzionato nei documenti scritti dal X secolo (Benericetti, 2002b, p. 170). Le sue strutture vengono ricostruite almeno due volte per volere della famiglia Guidi: il primo intervento avviene nel 1167 (Tolosanus, Chronicon, p. 66); il secondo nel 1258 (Tonduzzi, 1675, p. 294). Nel XIX secolo viene rinvenuta una lapide all'interno di una struttura (probabilmente una cisterna interrata) posta a pochi metri dalla torre (Lega, 1886, pp. 170-171). Nel manufatto - oggi conservato presso la Sala Manfredi della Pinacoteca Comunale di Faenza (Sec. XIV, Impresa araldica di Astorgio I Manfredi. calcare, cm. 109x68, n. inv. 200) - è incisa la seguente iscrizione: «Il 13 febbraio 1378, questo castello fu edificato dal magnifico signore Astorgio dei Manfredi, signore di Faenza» (trad. it. Cavina, 1964, p. 254). Le analisi archeologiche condotte sulle strutture superstiti del castello sembrano confermare questa notizia. In primo luogo, grazie le indagini stratigrafiche hanno stabilito che l'impianto planovolumetrico della torre è sostanzialmente unitario e le modifiche riguardano unicamente gli ambienti interni. La pianta circolare con sperone «a becco» caratterizza la torre di Castel Gavone (XV secolo: Murialdo, 2004, p. 70) e quella di Rontana, ottenuta addossando, tra la fine del ' 400 e la prima metà del ' 500 , uno sperone a un precedente edificio circolare (Cirelli, 2010, p. 86). Questo tipo di pianta, però, ricorre in alcuni fortilizi francesi del XII e XIII secolo: Château Gaillard, Château de La Roche-Guyon e la Tour Blanche (Brown, 2004, p. 54). I caratteri murari della torre di Ceparano suggeriscono una datazione tardomedievale dell'impianto. Le sue strutture sono realizzate con elementi misti («da 

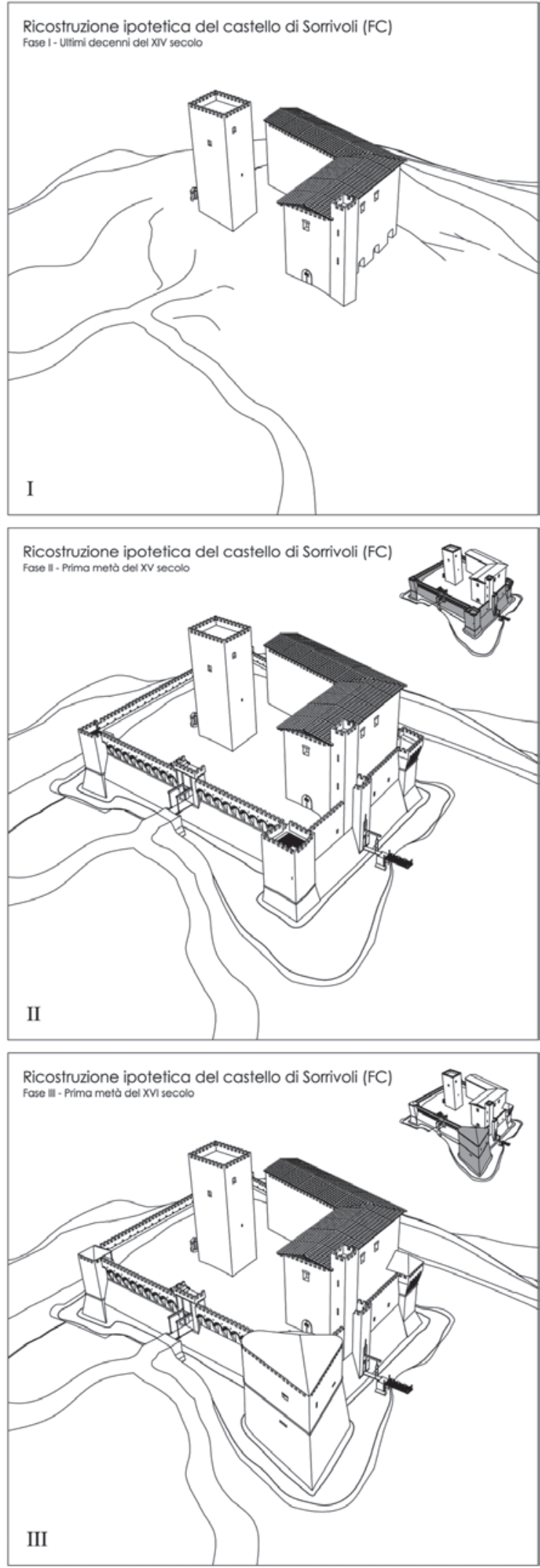

Fig. 15. Ipotesi di ricostruzione del castello di Sorrivoli (Roncofreddo, FC) in alcuni momenti della sua storia: I - ultimi decenni del XIV secolo; II prima metà del XV secolo; III - prima metà del XVI secolo (elaborazione grafica Andrea Fiorini, Emanuela Maroni, Alberto Urcia).
Sintesi storico-archeologica: la torre e $i$ magazzini trecenteschi. Nel 1392 esponenti della famiglia Malatesta invadono i territori dell'arcivescovo di Ravenna e conquistano il borgo fortificato di Sorrivoli. All'interno del castello, sulla collina più elevata, si costruiscono alcuni edifici per la raccolta delle derrate agricole e una torre quadrata (in materiali misti), con funzione abitativa e di controllo del territorio (fig. 15: I). Per progettare questo edificio vengono rispettate le normative comunali della città di Cesena (malatestiana dal 1378) mentre per la costruzione delle sue strutture murarie viene finanziata la ricerca, in aree molto distanti da Sorrivoli, di arenaria particolarmente resistente.

\section{Fase II (prima metà del xv secolo)}

A questa fase appartengono le strutture principali che qualificano il tipo architettonico della rocca: l'ingresso pedonale (CF7), le quattro torri angolari in materiali misti (CF2, CF8-10) e le relative cortine murarie. La datazione di queste strutture si desume dai caratteri costruttivi e formali di alcuni elementi architettonici. In primo luogo, una cordonatura in laterizi sagomati, con profilo a quarto di cerchio convesso, disposti a formare un cordone compreso fra due filari di mattoni, nell'insieme di medio spessore. Cordonature simili si trovano nella rocca malatestiana di Montefiore Conca (prima metà del XV secolo) e nelle torri quattrocentesche della cinta urbana di Cesena. Altri importanti indicatori cronologici sono alcune feritoie da tiro - in particolare quelle per l'utilizzo di armi da fuoco caratterizzate da una scudatura litica a foro tondo (tipo A6) e quelle con camera di manovra e luce «a finestrella» (tipo A1) - che collocano queste strutture nella prima metà del Quattrocento (fig. 16). La rocca, in effetti, doveva essere già stata completata nel 1448 se Malatesta Novello poté cederla a Pietro Roverella vicario della chiesa ravennate: arcis [...] scituate in dicto castro Sorivole (Fabbri,

scalpellino" e laterizi): i primi squadrati tramite attenta sbozzatura, spianati, di dimensioni eterogenee, disposti in filari irregolari, su letti di calce di spessore variabile; gli altri, ridotti in frammenti, su letti di calce di spessore variabile. Il materiale è di reimpiego, lo provano una serie di indizi che coesistono in quasi tutti gli elementi del campione murario esaminato: una consunzione disomogenea della superficie lapidea, la rottura parziale degli spigoli e tracce grossolane di strumenti a punta che "tagliano» una finitura già conclusa. Questa tecnica muraria è documentata in Liguria (atlante cronotipologico ISCUM) dal XIII secolo fino al Quattrocento (Pesce, 2009). Anche gli elementi architettonici della torre rimandano a una prassi fortificatoria tardomedievale: il cordolo litico, che appare ben ammorsato alla muratura e non sembra di reimpiego, marca il confine tra la scarpa e il paramento verticale. Questo manufatto compare nelle fabbriche fortificate dalla prima metà XIV secolo in concomitanza con la diffusione delle armi da fuoco (Luisi, 1996, p. 110; Taddei, 2004) e si afferma nel XV secolo (Martini, Trattati, II, passim). 


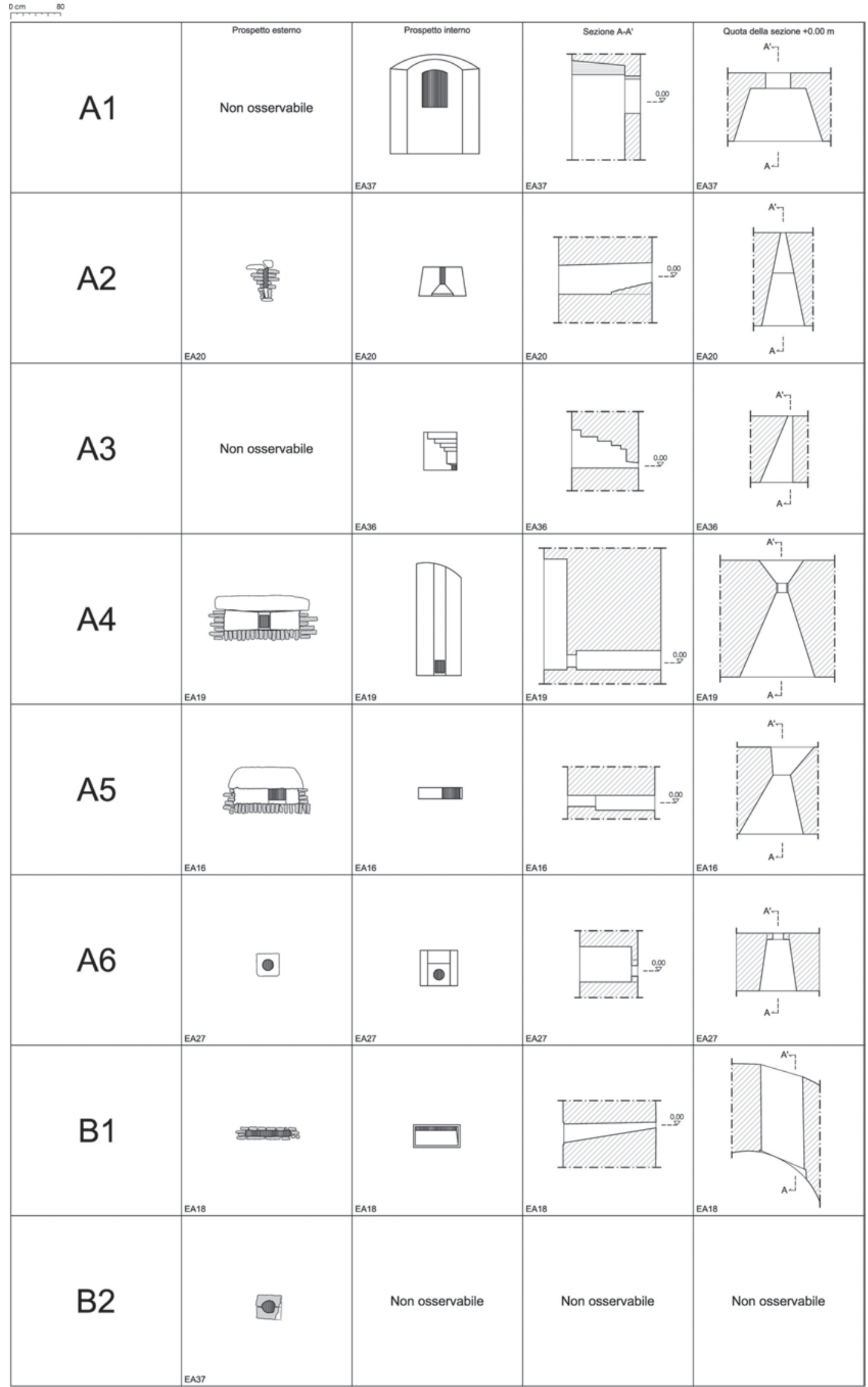

Fig. 16. Tipi di feritoie individuate nel castello di Sorrivoli (indagine a cura di Emanuela Maroni). 
Falcioni, Riva, 2003, p. 323). Dallo stesso documento si apprende, inoltre, che la rocca era munita di un fossato: castri Sorivole scituate in dicto castro Sorivole iuxta fossatum dicte arcis iuxta vias comunis dicti castri et iuxta ripas dicte arcis.

Prima di realizzare la rocca di Sorrivoli le maestranze malatestiane sono impegnate pochi chilometri più a Nord, a San Giorgio di Cesena (Conti, 1983). Della fortezza militare voluta da Andrea Malatesta oggi non rimane nulla ma i documenti iconografici, cartografici e fotografici ci mostrano un complesso architettonico a pianta quadrangolare, con superficie d'ingombro di circa 10000 metri quadri, munito di torri angolari simili a quelle della rocca di Sorrivoli. Sempre nel XV secolo a 5 chilometri dal castello di Sorrivoli, presso il castello di Monteleone, si apre un altro grande cantiere edilizio (Brunetti, 2003). Qui, attorno a una torre quadrangolare di XIII secolo, i Malatesti fanno costruire una fortezza in materiali misti (litici e laterizi). Negli angoli le maestranze malatestiane realizzano torri simili nella tecnica costruttiva e nella forma a quelle presenti nel fronte Sud della rocca di Sorrivoli (CF9-10).

Sintesi storico-archeologica: la rocca quattrocentesca. Nella prima metà del XV secolo la Chiesa di Roma riconosce ai Malatesti il controllo di Sorrivoli e di altri castelli arcivescovili. Si decide, pertanto, di aprire un grande cantiere edilizio per realizzare una sede adeguata al potere oramai consolidato di questa famiglia. Ai piedi del nucleo originario trecentesco si scava un largo fossato difensivo e lungo i fianchi della collina si addossano quattro torri angolari in materiali misti (litici e laterizi) collegate da altrettante cortine murarie (fig. 15: II). Mentre si eseguono questi importanti lavori la signoria territoriale dei Malatesti si popola di altri edifici simili. A Monteleone viene allestito un complesso architettonico fortificato attorno al nucleo preesistente mentre a Cesena si costruiscono le torri quadrangolari della cinta urbana dotandole dello stesso tipo di cordonatura riscontrabile nelle torri angolari della rocca di Sorrivoli. Nel frattempo Sigismondo Pandolfo Malatesta promuove importanti lavori di ampliamento della rocca di Montefiore Conca $(\mathrm{RN})$. Anche in questo caso alcune torri della cinta più esterna presentano la stessa cordonatura individuata a Cesena e Sorrivoli.

\section{Fase III (prima metà del xvı secolo)}

A questa fase appartiene il grande baluardo (CF3) che ingloba la torre d'angolo Nord-Ovest della rocca (CF2). La sua pianta, «ad asso di picche», ricorre nell'architettura militare del XVI secolo ${ }^{12}$. Allo stesso periodo rimandano le analisi tipologiche delle aperture di questo imponente edificio: feritoie da tiro «alla francese» (tipi A4, A5) (Palloni, 2000, p. 40; Palloni, 1995, p. 27).

Sintesi storico-archeologica: la soluzione al crescente potere distruttivo delle artiglierie, il grande bastione cinquecentesco. Nella prima metà del XVI secolo la maggior efficacia delle artiglierie assedianti non è più trascurabile e $\mathrm{i}$ Roverella - ai quali era stata concessa la contea di Sorrivoli - decidono di aggiornare i sistemi difensivi e offensivi della rocca malatestiana promuovendo la costruzione di un bastione (in laterizio) appuntito in direzione della strada di accesso al castello e dotato di grandi aperture per armi da fuoco (fig. 15: III). Nello stesso secolo altri edifici di questo tipo vengono innalzati in Romagna e nel resto dell'Italia. A pochi chilometri di distanza da Sorrivoli, ad esempio, il lato Ovest del borgo fortificato di Saludecio (RN) è sorvegliato da una struttura che presenta la stessa pianta «ad asso di picche».

\section{Fase IV (xVII secolo)}

Appartengono a questa fase una serie di strutture realizzate per ampliare lo spazio abitativo della fortezza. La cortina Nord della rocca viene soppiantata da un lungo e alto corpo di fabbrica a pianta rettangolare (CF4). Nell'ambito di questo intervento si innalza la torre angolare Nord-Est (CF8) e si restaurano le strutture sommitali del grande bastione (CF3) e della torre angolare Sud-Est (CF9). In questa fase, infine, rientra una porta del mastio (CF1), realizzata operando un taglio nel muro Nord dell'edificio e che serviva, probabilmente, per accedere al nuovo palazzo (CF4).

Per questa fase edilizia non è possibile stabilire una datazione precisa. In base ai rapporti di anteriorità/posteriorità con i manufatti di cronologia certa (appartenenti alle altre fasi) è possibile ottenere solamente indicazioni di cronologia relativa. Nonostante ciò, la riconversione a uso abitativo dei vecchi castelli medievali è un fenomeno piuttosto documentato in età moderna (secc. XVI-XVIII) e soprattutto a partire dal XVII secolo. Ad esempio, molti

\footnotetext{
${ }^{12}$ La pianta del bastione angolare (CF3) presenta una forma «ad asso di picche» molto simile a quella di alcuni corpi di fabbrica cinquecenteschi inseriti nelle mura della città di Ferrara (Di Fabio, 2003, pp. 56-59, 169) e di Urbino (LuNI, Ermeti, 1997, pp. 41-50). Un altro esempio di bastione "ad asso di picche» si trova a pochi chilometri di distanza da Sorrivoli, nel castrum Sancti Laudicii (Saludecio, RN). La struttura, che protegge il lato Ovest del borgo fortificato, viene datata agli inizi del XVI secolo (Tosi Brandi, 2007, pp. 167). Altre strutture simili si trovano nella fortezza di Castellamare a Palermo (prima metà del xvi secolo). Anche qui, come a Sorrivoli, i baluardi inglobano torri angolari più antiche (Santoro, 2001, pp. 339-342).
} 
castelli rurali della provincia di Piacenza, vengono trasformati in edifici residenziali quando si raggiunge una certa stabilità politica, vale a dire, sotto il dominio della famiglia dei Farnese (seconda metà del XVII secolo) ${ }^{13}$.

Considerando tutti i dati a disposizione si avanza per la fase edilizia IV una collocazione cronologica prossima al XVII secolo.

Sintesi storico-archeologica: la riconversione in palazzo residenziale e il restauro settecentesco. Nel XVII secolo la rocca viene trasformata in comoda residenza di campagna attraverso una serie di interventi di altissimo impatto strutturale. In Italia la riconversione a uso abitativo delle vecchie fortificazioni medievali è un fenomeno piuttosto frequente che si diffonde a partire dall'età moderna. Nella seconda metà del XVII secolo, ad esempio, i coronamenti merlati del castello piacentino di Zena vengono rimossi, le sue murature foderate e innalzate (Fabbri, Fiorini, Gualtieri, c.s.).

\section{Le altre fasi}

Ricostruito il portone d'ingresso alla fine del XVII secolo (fase V), nel Settecento (fase VI) vengono irrobustite le strutture della rocca per garantire un adeguato sostegno ai nuovi piani abitativi. Alte scarpe basamentali vengono addossate ai paramenti murari ottenendo, così, l'ispessimento di tutte le sezioni murarie. L'aspetto attuale del complesso architettonico è dovuto, in buona sostanza, ai lavori realizzanti nell'ambito di questo importante cantiere di restauro.

\section{Bibliografia}

Archetti, V.; Fiorini, A., c.s., Fotomodellazione e stereofotogrammetria per la creazione di modelli stratigrafici in archeologia dell'architettura, "Archeologia e Calcolatori».

Artocchini, C., 1997, L'architettura castrense nel contado piacentino, in AA.VV., Storia di Piacenza, III, Dalla signoria viscontea al principato farnesiano (13131545), Piacenza, pp. 655-688.

Augenti et alii, 2009a = Augenti, A.; Fiorini, A.; Montanari, M.; Sericola, M.; Urcia, A.; Zaffagnini F., Archeologia dell'architettura in Emilia-Romagna: primi passi verso un progetto organico, "Ocnus», 17, 2009, pp. 65-76.

Augenti et alii, 2009b = Augenti, A.; Cirelli, E.; Fiorini, A.; Ravaioli, E., L'incastellamento in Romagna: indagini 2006-2008, in Favia P., Volpe G. (a cura di), $V$ Congresso Nazionale di Archeologia Medievale (Palazzo della Dogana, Salone del Tribunale, Foggia; Palazzo dei Celestii, Auditorium, Manfredonia; 30 settembre-3 ottobre 2009), Firenze 2009, pp. 341-348.

Augenti et alii, 2010 = Augenti, A.; Fiorini, A.; Cirelli, E.; Ravaioli, E., Insediamenti e organizzazione del territorio in Romagna (secoli X-XIV), "Archeologia Medievale», XXXVII, 2010, pp. 61-92.

Benericetti R. (a cura di), 2002a, Le carte ravennati del decimo secolo. Archivio arcivescovile, III, (aa. 976-999), Bologna.

Benericetti R. (a cura di), 2002b, Le carte ravennati del decimo secolo nell'archivio arcivescovile di Ravenna, II, (aa. 957-976), Imola.

${ }^{13}$ Su questo argomento v.: Artocchini, 1997, pp. 657-659; Coccioli Mastroviti, 1990, p. 93; Matteucci, Manfredi, Coccioli Mastroviti, 1991, pp. 14-19.
Bertacci et alii, 1972 = Bertacci, L.; Degli Esposti, V.; Foschi, M.; Venturi S. (a cura di), Territorio e conservazione. Proposta di rilevamento dei beni culturali immobili nell'Appennino bolognese, Bologna 1972.

Bianchi, G., 1996, Trasmissione dei saperi tecnici e analisi dei procedimenti costruttivi, "Archeologia dell'Architettura», I , pp. 53-64.

Bolognesi, G.; Lorenzini, E., 2004, Storia di Sorrivoli, [s.l.].

Braschi, G. B., 1738, Memoriae Caesenates sacrae, et profanae [...], Roma.

Brogiolo, G. P., 1988, Archeologia dell'edilizia storica, Como.

Brogiolo, G. P., 2002, L'archeologia dell'architettura in Italia nell'ultimo quinquennio (1997-2001), "Arqueología de la Arquitectura», 1, 19-26.

Brown, R. A., 2004, Allen Brown's English castles, Woodbridge.

Brunetti, G., 2003, Il restauro del castello di Monteleone (FO). Storia, materiali e tecniche costruttive di un castello malatestiano, Università degli Studi di Ferrara, Facoltà di Architettura, rel. A. Ugolini, R. Fabbri, A.A. 2002-2003.

Cagnana, A., 1996, Le tecniche murarie nelle valli del Ceresio: evidenze archeologiche e problemi interpretativi, in Della Torre S., Mannoni T., Pracchi V. (a cura di), Magistri d'Europa: eventi, relazioni, strutture della migrazione di artisti e costruttori dai laghi lombardi, Milano, pp. 447-459.

Cavina, G., 1964, Antichi fortilizi di Romagna: valle del Lamone e del Marzeno, valle del Sintria e del Senio, Brisighella, Faenza.

Cirelli, E., 2009, Lo scavo del castello di Rontana, in Augenti et alii, 2009b, pp. 346-347.

Cirelli, E., 2010, Il castello di Rontana, in Augenti et alii, 2010, pp. 85-89.

Coccioli Mastroviti, A., 1990, La grande decorazione nelle ville della campagna piacentina: primi risultati da una indagine. Il Settecento, "Strenna Piacentina», pp. $93-103$.

Conti, G., 1983, Ville fortificate dell'agro cesenate in età malatestiana, "Studi Romagnoli», XXXIV, pp. 459-497.

De Luca, L., 2011, La fotomodellazione architettonica. Rilievo, modellazione, rappresentazione di edifici a partire da fotografie, Palermo.

De Maio, F., 2007, L'edilizia civile in pietra nella città di Grosseto: i prospetti di via Aldobrandeschi, in Citter C. (a cura di), Archeologia Urbana a Grosseto. II. Edizione degli scavi urbani 1998-2005, Borgo San Lorenzo (Firenze), pp. 262 270.

Di Fabio, M. R. (a cura di), 2003, Le mura di Ferrara. Storia di un restauro, Argelato.

Docci, M.; Maestri, D., 1984, Il rilevamento architettonico. Storia, metodi e disegno, Roma-Bari.

Docci, M.; Maestri, D., 1992, Manuale di rilevamento architettonico e urbano, Roma Bari.

Doglioni, F., 1997, Stratigrafia e restauro. Tra conoscenza e conservazione dell'architettura, Trieste.

Fabbri, P. G.; Falcioni, A.; Riva, C., 2003, Appendici, in Fabbri P.G., Falcioni A. (a cura di), La signoria di Malatesta Novello Malatesti (1433-1465), Rimini, pp. 293-448.

Fabbri, P. G., 2003, Gli aspetti politici, militari, economici ed istituzionali della signoria di Malatesta Novello, in Fabbri P.G., Falcioni A. (a cura di), La signoria di Malatesta Novello Malatesti (1433-1465), Rimini, pp. 29-198.

Fabbri, B.; Fiorini, A.; Gualtieri, S., c.s., Il castello di Zena (PC): storia, archeologia e archeometria, "Archeologia dell'Architettura".

Fantuzzi, Monumenti = M. Fantuzzi, Monumenti ravennati de' secoli di mezzo per la maggior parte inediti, I-VI, Venezia 1801-1804.

Ferrando Cabona, I.; Crusi, E., 1981, Storia dell'insediamento in Lunigiana. Valle del Rosaro, Genova.

Ferrando Cabona, I.; Crusi, E., 1988, Storia dell'insediamento in Lunigiana. Alta Valle Aulella, Genova.

Fiorani, D., 2004, Lo studio della geometria e delle dimensioni, in Restauro architettonico e strumento informatico, Napoli, pp. 48-49.

Fiorini, A., 2008, Esperienze di fotomodellazione e stereofotogrammetria archeologi$c a$, in Volpe G., De Felice G., Sibilano M.G. (a cura di), L'Informatica e il metodo della stratigrafia, Atti del Workshop (Foggia, 6-7 giugno 2008), Bari, pp. 175-186.

Fiorini, A., 2009, I castelli della Romagna: le tecniche edilizie, in Augenti et alii, 2009b, pp. 343-346.

Fiorini, A., 2010a, I castelli della Romagna: le tecniche edilizie, le maestranze, i committenti, in Augenti et alii 2010, pp. 75-85. 
Fiorini, A., 2010b, Archeologia dell'architettura in Romagna: progetto per la realizzazione di un atlante delle tecniche costruttive di età medievale, Università di Bologna, Tesi di Dottorato di Ricerca in Archeologia, XXII ciclo.

Fiorini, A. (a cura di), c.s., Il castello di Sorrivoli (Roncofreddo, Forli-Cesena): storia $e$ archeologia dell'architettura, Bologna.

Francovich, R.; Parenti, R. (a cura di), 1988, Archeologia e restauro dei monumenti, I Ciclo di Lezioni sulla Ricerca applicata in Archeologia (Certosa di Pontignano 1987), Firenze.

Gelichi, S., 1997, Note sul ritrovamento di Sorrivoli, in S. Gelichi (a cura di), Ceramiche tardo-medioevali a Cesena, Cesena, pp. 83-100.

Ghiretti, A.; Ottoni, F., 2009, Analisi grafica e metrologia, in Giandebiaggi, P.; Mambriani, C.; Ottoni, F. (a cura di), Santa Maria del Quartiere in Parma. Storia, rilievo e stabilità di una fabbrica farnesiana, Parma, pp. 174-185.

Giovannini, P.; Parenti, R., 2006, Torre Vanga a Trento. Aspetti metodologici e operativi dell'analisi stratigrafica finalizzata al cantiere di restauro, "Archeologia dell'Architettura", XI, pp. 69-86.

Lega, A., 1886, Fortilizi in Val di Lamone, Faenza (rist. anast. Sala Bolognese 1989).

Luisi, R., 1996, Scudi di pietra. I castelli e l'arte della guerra tra Medioevo e Rinascimento, Bari.

Luni, M.; Ermeti, A.L., 1997, Le mura di Urbino tra tardoantico e medioevo, in Gelichi, S. (a cura di), Atti del I Congresso Nazionale di Archeologia Medievale (Pisa 29-31 maggio 1997), Firenze, pp. 41-50.

Luschi, C. M. R., 2006, Le misure dei castelli: il caso di Prato, le verifiche di Manfredonia e la riconoscibilità di una matrice geometrico compositiva nella pieve castello di Stazzema, in Manenti Valli F. (a cura di), Le misure del castello. Un percorso per la conoscenza dell'architettura fortificata, Atti del convegno nazionale di studi castellologici (13-14 ottobre 2006), Roma, pp. 32-38.

Mannoni T., 1976, L'analisi delle tecniche murarie medievali in Liguria, in Atti del Colloquio internazionale di archeologia medievale (Palermo-Erice, 20-22 settembre 1974), Palermo, pp. 291-300 (ora anche in Mannoni T., Caratteri costruttivi dell'edilizia storica, Genova 1994, pp. 7-18).

Mannoni, T., 1997, Il problema complesso delle murature storiche in pietra 1. Cultura materiale e cronotipologia, "Archeologia dell'Architettura», II, pp. 15-24.

Mannoni, T., 2005, Archeologia della produzione architettonica. Le tecniche costruttive, "Arqueología de la Arquitectura", IV, pp. 11-19.

Martini, Trattati = F. di Giorgio Martini, Trattati di Architettura, Ingegneria e Arte Militare, a cura di Maltese C., I-II, Milano 1967.

Matteucci, A. M.; Manfredi, C. E.; Coccioli Mastroviti, A., 1991, Ville Piacentine, Piacenza.

Mazzatinti, G. (a cura di), 1909, Annales Forolivienses ab origine urbis usque ad annum MCCCCLXXIII, in Rerum Italicarum Scriptores, XXII/2, Città di Castello.

Montalti, P. (a cura di), 1986, La cinta muraria di Cesena, Modena.

Murialdo, G., 2004, Castel Gavone e la trasformazione di un castello medievale tra Quattro e Cinquecento, "Ligures», II, pp. 59-78.

Palloni, D., 1995, La Transizione, in Rocche e Bombarde fra Marche e Romagna nel XV secolo, a cura di M. Mauro, Ravenna, pp. 13-46.

Palloni, D., 2000, Evoluzione delle bombardiere, "Castellum», 42, pp. 33-42.

Parenti, R., 1987, Una proposta di classificazione tipologica delle murature postclassiche, in Biscottin, G.; Angeletti, R. (a cura di), Conoscenze e sviluppi teorici per la conservazione di sistemi costruttivi tradizionali in muratura, Atti del convegno di Studi (Bressanone 23-26 Giugno 1987), Padova, pp. 49-61.

Parenti, R., 2000, s.v. Architettura, archeologia della, in Francovich, F.; Manacorda, D. (a cura di), Dizionario di archeologia. Temi, concetti e metodi, RomaBari, pp. 39-43.
Pasini, G. (a cura di), 2002, Malatesta Novello Magnifico Signore. Arte e cultura di un principe del Rinascimento, San Giorgio di Piano.

Pasquali, G., 1996, Una signoria rurale assente o silente? Il caso anomalo della Romagna, in Spicciani A., Violante C. (a cura di), La signoria rurale nel Medioevo italiano, I, Pisa, pp. 63-80.

Perogalli, C., 1999, Brancaleone a Ravenna. Rocca capostipite, in Mauro, M. (a cura di), La rocca di Ravenna (Rocca Brancaleone), Ravenna, pp. 63-77.

Pesce, G., 2009, [Lettera personale dell'autore].

Quirós Castillo, J. A., 1992, Cronotipologia di portali nell'alta Valdinievole: la montagna pesciatina (PT), «Archeologia Medievale», XIX, pp. 729-737.

Rabotti, G., 1993, Dai vertici dei poteri medievali: Ravenna e la sua Chiesa fra diritto e politica dal X al XIII secolo, in Vasina A. (a cura di), Storia di Ravenna, III, Dal Mille alla fine della Signoria polentana, Venezia, pp. 129-168.

Riva, C., 1985, Città e territorio in epoca malatestiana: contributo per una analisi topografica, in Vasina A. (a cura di), Storia di Cesena, II, 2, Il Medioevo (secoli $X I V-X V)$, Rimini, pp. 257-302.

Robertson, J., 1985, Cesena: governo e Società dal sacco dei Bretoni al dominio di Cesare Borgia, in Vasina A. (a cura di), Storia di Cesena, II, 2, Il Medioevo (secoli $X I V-X V)$, Rimini, pp. 5-92.

Rossi, G., 1589, Historiarum Rauennatum libri decem, Venezia.

Salvatori, M., 2006, Manuale di metrologia. Per architetti studiosi di storia dell'architettura e archeologi in Italia, Napoli.

Sansovino, F., 1609, Della origine, et de' fatti delle famiglie illustri d'Italia, Venezia.

Santoro, R., 2001, Palermo, Castello a Mare, in AA.VV., Castelli medievali di Sicilia. Guida agli itinerari castellani dell'isola, Palermo, pp. 339-342.

Sassi, M., 2005, Castelli in Romagna, Cesena.

Scardino, L., 2000, Tra Cesena e Ferrara. La quadreria dei conti Roverella, «Romagna arte e storia», 58, pp. 91-100.

Taddei, D., 2004, s.v. cordolo, in Taddei D. (a cura di), Le parole del castello. Nomenclatura castellana, Sesto Fiorentino, p. 85.

Tolosanus, Chronicon $=$ Tolosanus, Magistri Tolosani Chronicon Faventinum: (AA. 20 av. C.-1236), a cura di Giuseppe Rossini, in Rerum Italicarum Scriptores, n.s., XXVIII/1, Bologna 1936-1939.

Tonduzzi, G. C., 1675, Historie di Faenza, Faenza (rist. fotomec. Bologna 1967).

Tosi Brandi, E., 2007, Castelli e tombe della Provincia di Rimini, in Tosi Brandi E. (a cura di), Castelli e fortificazioni del riminese, Rimini, pp. 74-185.

Urcia, A., 2009, Il Castello di Sorrivoli (FC): analisi archeologica dellelevato, Corso di laurea in Beni Archeologici, Facoltà di Conservazione dei Beni Culturali, Università di Bologna, A.A. 2008-2009, relatore prof. Andrea Augenti.

Vasina, A., 1983, La città e il territorio prima e dopo il Mille, in Vasina A. (a cura di), Storia di Cesena, II, 1, Il Medioevo (secoli VI-XIV), Rimini, pp. 75-182.

Vasina, A., 1986, Comuni e signorie in Emilia e in Romagna, Torino.

Veggiani, A., 1968, Rilevamento geologico delle cave malatestiane nel territorio cesenate, «Studi Romagnoli», XIX, pp. 343-367.

Zaffagnini, F., 2009, Geologia applicata all'archeologia dei castelli medievali: l'indagine presso $i$ siti di Castelnuovo (FC), Sorrivoli (FC), Ceparano(RA), Giaggiolo (FC), in Augenti et alii, 2009a, pp. 68-71.

Zupko, R. E., 1997, s.v. Metrologia architettonica, in Enciclopedia dell'Arte Medievale. Treccani, VIII, Roma, pp. 354-357.

Recibido: 31 de mayo de 2011 Aceptado: 11 de agosto de 2011 